\title{
Approximations of Lipschitz maps via immersions and differentiable exotic sphere theorems ${ }^{* \dagger}$
}

\author{
Kei KONDO $¥$. Minoru TANAKA
}

July 30, 2019

\begin{abstract}
As our main theorem, we prove that a Lipschitz map from a compact Riemannian manifold $M$ into a Riemannian manifold $N$ admits a smooth approximation via immersions if the map has no singular points on $M$ in the sense of F.H. Clarke, where $\operatorname{dim} M \leq \operatorname{dim} N$. As its corollary, we have that if a bi-Lipschitz homeomorphism between compact manifolds and its inverse map have no singular points in the same sense, then they are diffeomorphic. We have three applications of the main theorem: The first two of them are two differentiable sphere theorems for a pair of topological spheres including that of exotic ones. The third one is that a compact $n$-manifold $M$ is a twisted sphere and there exists a bi-Lipschitz homeomorphism between $M$ and the unit $n$-sphere $S^{n}(1)$ which is a diffeomorphism except for a single point, if $M$ satisfies certain two conditions with respect to critical points of its distance function in the Clarke sense. Moreover, we have three corollaries from the third theorem; the first one is that for any twisted sphere $\Sigma^{n}$ of general dimension $n$, there exists a bi-Lipschitz homeomorphism between $\Sigma^{n}$ and $S^{n}(1)$ which is a diffeomorphism except for a single point. In particular, there exists such a map between an exotic $n$-sphere $\Sigma^{n}$ of dimension $n>4$ and $S^{n}(1)$; the second one is that if an exotic 4sphere $\Sigma^{4}$ exists, then $\Sigma^{4}$ does not satisfy one of the two conditions above; the third one is that for any Grove-Shiohama type $n$-sphere $N$, there exists a bi-Lipschitz homeomorphism between $N$ and $S^{n}(1)$ which is a diffeomorphism except for one of points that attain their diameters.
\end{abstract}

\section{Introduction}

\subsection{Motivations and our main theorem}

We first mention our motivations behind our purpose, which are exotic structures: No one needs the introduction of exotic $n$-dimensional spheres $\Sigma^{n}$, however $\Sigma^{n}$ were very first

${ }^{*} 2010$ Mathematics Subject Classification: Primary 49J52, 53C20; Secondary 57R12, 57R55.

${ }^{\dagger}$ Key words and phrases: bi-Lipschitz homeomorphism, differentiable sphere theorem, exotic spheres, Lipschitz map, non-smooth analysis, smooth approximation.

${ }^{\ddagger}$ Partly supported by the two Grant-in-Aids for Science Reserch (C), JSPS KAKENHI Grant Numbers $15 \mathrm{~K} 04846$ and 16K05133. 
discovered by Milnor [25] in the case of $n=7$, which is, by definition, homeomorphic to the standard $n$-sphere $S^{n}$ but not diffeomorphic to it. Note that the smooth 4 -dimensional Poincaré conjecture (SPC4), that is, the problem of the existence of an exotic structure on the 4-sphere, is still open.

Due to exotic structures, we always have technical difficulties when investigating whether a topological sphere theorem can be reinforced into a differentiable sphere theorem. The difficulties become clearer from global Riemannian geometry's stand point: It follows from Smale's h-cobordism theorem [35] together with [34] that every homotopy sphere of dimension $n \geq 5$ is a twisted sphere, which is a smooth manifold obtained by glueing two standard $n$-discs along their boundaries under a boundary diffeomorphism. This implies that every $\Sigma^{n}(n>4)$ is actually twisted, since $\Sigma^{n}$ is a homotopy sphere. Applying Weinstein's deformation technique for metrics ([37, Proposition C]) to both of two discs embedded smoothly into a twisted sphere $X$ of general dimension, we see that $X$ admits a metric such that the cut locus 1 of some point on $X$ is a single point. By all together above, we have

Theorem 1.1 (Also see [2, Proposition 7.19]) Every exotic sphere $\Sigma^{n}$ of dimension $n>4$ admits a metric such that there is a point whose cut locus consists of a single point.

Thus, it is very difficult for us to notice the difference between $\Sigma^{n}$ and $S^{n}$ from the point of view of two exponential maps at the single point on $\Sigma^{n}$ and at any point on $S^{n}$. For example, one of open problems in global Riemannian geometry is if a Grove-Shiohama type $n$-sphere can be diffeomorphic to $S^{n}$. Here, a complete Riemannian manifold $V$ is called a Grove-Shiohama type sphere if sectional curvature $K_{V} \geq 1$ and its diameter $\operatorname{diam}(V)>\pi / 2([18])$. Since such a $V$ is twisted, from Theorem 1.1 we can infer that some single cut point on $V$ is a big obstacle whenever approximating a homeomorphism, in fact which is bi-Lipschitz, between $V$ and $S^{n}$ by diffeomorphisms.

Hence, there is a cardinal importance to do an analysis of such singular points on an arbitrary manifold. For this, we employ a notion used in non-smooth analysis of F.H. Clarke ([5], 6]), i.e., a non-singular point for a Lipschitz function/map in this article. The following example shows that the non-smooth analysis is a strong tool in differential geometry.

Example 1.2 Let $M$ be a complete Riemannian manifold, $d$ the distance function of $M$. Take any point $p \in M$, and fix it. Set $d_{p}(x):=d(p, x)$ for all $x \in M$. Then, the point $q \in M \backslash\{p\}$ is a critical point of $d_{p}$ (or critical point for $p$ ) in the sense of Grove-Shiohama [18], if for every nonzero tangent vector $v \in T_{q} M$ at $q$, there exists a minimal geodesic segment $\gamma$ emanating from $q$ to $p$ such that

$$
\angle(v, \dot{\gamma}(0)) \leq \frac{\pi}{2}
$$

\footnotetext{
${ }^{1}$ The cut locus $\operatorname{Cut}(p)$ of a point $p$ in a complete Riemannian manifold $M$ is, by definition, the closure of the set of all points $x \in M$ such that there are at least two minimal geodesics emanating from $p$ to $x$. Then, a point in $\operatorname{Cut}(p)$ is called the cut point of $p$. For example, $\operatorname{Cut}(N)$ of $N:=(0, \ldots, 0,1) \in S^{n}(1)$ is $\{(0, \ldots, 0,-1)\}$, where $S^{n}(1):=\left\{v \in \mathbb{R}^{n+1} \mid\|v\|=1\right\}$. Note that the distance function from a point $x$ of $M$ is not differentiable at the cut point of $x$.
} 
Here, $\angle(v, \dot{\gamma}(0))$ denotes the angle between $v$ and $\dot{\gamma}(0):=(d \gamma / d t)(0)$. Note that a critical point of $d_{p}$ is the cut point of $p$. Assume that, for some $r>0, \partial B_{r}(p):=\{x \in M \mid d(p, x)=$ $r\}$ has no critical points of $d_{p}$. By Gromov's isotopy lemma [15], $\partial B_{r}(p)$ is a topological submanifold of $M$. Since $\partial B_{r}(p)$ is also free of critical points of $d_{p}$ in the sense of Clarke (See Example 1.9), it follows from Clarke's implicit function theorem [7] that

in fact, $\partial B_{r}(p)$ is a Lipschitz submanifold of $M$.

We are going to give the definition of the Clarke sense later (See Definition 1.5] in Sect 1.2).

Our purpose of this article is to establish an approximation method for a Lipschitz map via diffeomorphisms using the notion of non-smooth analysis and to apply this method to prove differentiable sphere theorems. That is, our main theorem is as follows:

Theorem 1.3 (Main Theorem) Let $F: M \longrightarrow N$ be a Lipschitz map from a compact Riemannian manifold $M$ into a Riemannian manifold $N$, where $\operatorname{dim} M \leq \operatorname{dim} N$. If $F$ has no singular points on $M$ (in the sense of Clarke), then for any $\eta>0$, there exists a smooth immersion $f_{\eta}$ from $M$ into $N$ such that

$$
\max _{x \in M} d_{N}\left(F(x), f_{\eta}(x)\right)<\eta, \quad \operatorname{Lip}\left(f_{\eta}\right) \leq \operatorname{Lip}(F)(1+\eta) .
$$

Here, $\operatorname{Lip}\left(f_{\eta}\right)$ and $\operatorname{Lip}(F)$ denote the Lipschitz constants of $f_{\eta}$ and $F$, respectively, i.e.,

$$
\operatorname{Lip}\left(f_{\eta}\right):=\sup \left\{\frac{d_{N}\left(f_{\eta}(x), f_{\eta}(y)\right)}{d_{M}(x, y)} \mid x, y \in M, x \neq y\right\}
$$

and

$$
\operatorname{Lip}(F):=\sup \left\{\frac{d_{N}(F(x), F(y))}{d_{M}(x, y)} \mid x, y \in M, x \neq y\right\}
$$

where $d_{M}$ and $d_{N}$ are the distance functions of $M$ and $N$, respectively.

Remark 1.4 We give three remarks on Theorem 1.3.

- The definition of a singular point for a Lipschitz map, i.e., non-smooth analysis of Clarke, is given in Sect 1.2 .

- It should be emphasized that the reason why Clarke introduced the notion of nonsmooth analysis was not for approximations of a Lipschitz map via diffeomorphisms, but for the inverse function theorem for a Lipschitz map, which contains the classical one as a special case. See also Example 1.8 below.

- Our approximation method for Lipschitz maps to prove Theorem 1.3 generalizes the whole Grove-Shiohama one in [18]. See Sect.2.

As an indirect corollary of Theorem 1.3, for any twisted sphere $\Sigma^{n}$ of any dimension $n$, we can construct a concrete bi-Lipschitz homeomorphism $F$ between $\Sigma^{n}$ and $S^{n}$ which is a diffeomorphism except for a single point (See Corollary 1.15). Therefore, by this result together with Theorem 1.1, the existence of exotic $n$-spheres $(n>4)$ implies that we cannot approximate $F$ by diffeomorphisms. Hence, we must give careful consideration to 
sufficient conditions for a pair of topological spheres admitting a single cut point of some point, hence even for that of exotic ones, to be diffeomorphic.

As applications of Theorem 1.3, we prove differentiable sphere theorems not only for such a pair above, but for a pair of exotic $n$-spheres $(n>4)$, where we give the sufficient conditions for each pair to be diffeomorphic. See Sect.1.3.

\subsection{Non-smooth analysis and a corollary of Theorem 1.3}

F.H. Clarke very first established the non-smooth analysis in [5] and [6]. It is a strong tool not only in the optimal control theory (cf. [8]), but also in differential geometry (e.g., [20], [30], and Example [1.2): Let $M, N$ be Riemannian manifolds, and let $F: M \longrightarrow N$ be a Lipschitz map. In the case of $N=\mathbb{R}$, let $f:=F: M \longrightarrow \mathbb{R}$. By Rademacher's theorem [29], there exists a set $E_{F} \subset M$ of measure zero such that the differential $d F$ of $F$ exists on $M \backslash E_{F}$. Then, for each point $x$, there exists a sequence $\left\{x_{i}\right\}$ of $x_{i} \in M \backslash E_{F}$ convergent to $x$, and hence we can define the generalized differential $\partial F(x)$ of $F$ at $x \in M$ as follows:

$$
\partial F(x):=\operatorname{Conv}\left(\left\{\lim _{i \rightarrow \infty} d F_{x_{i}} \mid d F_{x_{i}} \text { exists as } x_{i} \in M \backslash E_{F} \longrightarrow x\right\}\right),
$$

where "Conv $(\cdot)$ " means "convex hull". In the case of $N=\mathbb{R}$, we call $\partial f(x)$ the generalized gradient of $f$ at $x$, i.e.,

$$
\partial f(x):=\operatorname{Conv}\left(\left\{\lim _{i \rightarrow \infty} \nabla f\left(x_{i}\right) \mid \nabla f\left(x_{i}\right) \text { exists as } x_{i} \in M \backslash E_{f} \longrightarrow x\right\}\right) .
$$

Here $\nabla f$ denotes the gradient vector field of $f$. Note that both definitions above do not depend on atlases (e.g., [20]), and that $\partial F(x), \partial f(x)$ are compact convex sets.

Definition $1.5([5],[6])$ Let $M, N$ be the same as above, and $U \subset M$ an open set.

- A point $x \in U$ is said to be non-singular for a Lipschitz map $F: U \longrightarrow N$, if every element in $\partial F(x)$ is of maximal rank.

- A point $x \in U$ is said to be non-critical for a Lipschitz function $f: U \longrightarrow \mathbb{R}$, if

$$
o \notin \partial f(x)
$$

where $o$ denotes the zero tangent vector at $x$.

We give several examples of Definition 1.5 with a few related remarks.

Example 1.6 Consider two functions $f_{1}(x):=x^{2}, f_{2}(x):=x+2$ on $(-2,3)$. Define the Lipschitz function $f(x):=\max \left\{f_{1}(x), f_{2}(x)\right\}$ on $(-2,3)$, i.e.,

$$
f(x)= \begin{cases}x^{2} & \text { on }(-2,-1) \cup(2,3), \\ x+2 & \text { on }[-1,2] .\end{cases}
$$

Note that $f$ is not differentiable at $x=-1,2$. Since

$$
\lambda \frac{d f_{1}}{d x}(-1)+(1-\lambda) \frac{d f_{2}}{d x}(-1)=-3 \lambda+1
$$


for all $\lambda \in[0,1]$, we have $\partial f(-1)=[-2,1]$. As well as above, we have $\partial f(2)=[1,4]$. Since $0 \in \partial f(-1)$ and $0 \notin \partial f(2), x=-1$ is a critical point of $f$ and $x=2$ is not that of $f$. Note that $f(-1)=1$ is the minimum value of $f$.

Example 1.7 ([6, Remark 1]) Let $F: \mathbb{R}^{2} \longrightarrow \mathbb{R}^{2}$ be the Lipschitz map defined by $F(x, y):=(|x|+y, 2 x+|y|)$. Note that $F$ is not differentiable at $(x, y)=(0,0)$. Then,

$$
\begin{aligned}
\partial F(0,0) & =\operatorname{Conv}\left(\left\{\left(\begin{array}{ll}
1 & 1 \\
2 & 1
\end{array}\right),\left(\begin{array}{cc}
1 & 1 \\
2 & -1
\end{array}\right),\left(\begin{array}{cc}
-1 & 1 \\
2 & 1
\end{array}\right),\left(\begin{array}{cc}
-1 & 1 \\
2 & -1
\end{array}\right)\right\}\right) \\
& =\left\{\left(\begin{array}{ll}
s & 1 \\
2 & t
\end{array}\right)|| s|\leq 1,| t \mid \leq 1\right\} .
\end{aligned}
$$

Thus, $(0,0)$ is non-singular for $F$.

Example $1.8\left(\left[\underline{6}\right.\right.$, Lemmas 3,4]) Let $F: \mathbb{R}^{n} \longrightarrow \mathbb{R}^{k}$ be a map that is Lipschitz near some point $p \in \mathbb{R}^{n}$, where $n \leq k$. Assume that $p$ is non-singular for $F$. Then for any $u \in S^{n-1}(1), \partial F(p) u:=\{A u \mid A \in \partial F(p)\}$ is convex in $\mathbb{R}^{k}$ and $o \notin \partial F(p) u$. Hence, there exist a vector $v \in S^{k-1}(1)$ and a number $\delta(p)>0$ such that

$$
\langle A u, v\rangle \geq 2 \delta(p)
$$

for all $A \in \partial F(p)$. Moreover, by the definition of $\partial F(p)$, we may find a number $r(p)>0$ satisfying

$$
\langle A u, v\rangle \geq \delta(p)
$$

for all $A \in \partial F(q)$ and all $q \in B_{r(p)}(p):=\left\{x \in \mathbb{R}^{n} \mid\|p-x\|<r(p)\right\}$. Furthermore, one can prove that if $q_{1}, q_{2} \in \overline{B_{r(p)}(p)}:=\left\{x \in \mathbb{R}^{n} \mid\|p-x\| \leq r(p)\right\}, F$ is $\delta(p)$-expanding near $p$, that means that

$$
\left\|F\left(q_{2}\right)-F\left(q_{1}\right)\right\| \geq \delta(p)\left\|q_{2}-q_{1}\right\| .
$$

Using this inequality, Clarke proved the existence of a local Lipschitzian inverse at a non-singular point of a Lipschitz map (See [6, Theorem 1]).

Example 1.9 (Critical points of Grove-Shiohama [18]) Let $M$ be a complete Riemannian manifold, $d$ the distance function of $M$, and fix $p \in M$. Assume that $q \in M$ is not a critical point for $p$ in the sense of Grove-Shiohama (Refer to Example 1.2 for its definition). By definition, there exists $w \in T_{q} M \backslash\{o\}$ such that $\angle(w, \dot{\gamma}(0))>\pi / 2$ holds for all minimal geodesic segments $\gamma$ emanating from $q$ to $p$. Hence, $o \notin \partial d_{p}(q)$, where $d_{p}(x):=d(p, x)$ for all $x \in M$. Note that a point $q \neq p$ is a critical point of $d_{p}$ if and only if $o \in \partial d_{p}(q)$. Since $\partial d_{p}(p)$ equals the unit closed ball centered at the origin of $T_{p} M, o \in \partial d_{p}(p)$. Note that, even if $o \notin \partial d_{p}(q)$, it is possible to occur that $q$ is a cut point of $p$ in general.

As a direct consequence of Theorem 1.3, we have

Corollary 1.10 Let $F$ be a bi-Lipschitz homeomorphism from a compact Riemannian manifold $M$ onto a Riemannian manifold $N$. If $F$ and $F^{-1}$ have no singular points on $M$ and $N$, respectively, then $M$ and $N$ are diffeomorphic. 
Remark 1.11 Shikata would be the first researcher who approximated a bi-Lipschitz homeomorphism via diffeomorphisms: In [31, he introduced a distance for a pair of compact differentiable manifolds which are bi-Lipschitz homeomorphic and proved that if the distance between such a pair is smaller than a certain positive constant, then the bi-Lipschitz map can be approximated via diffeomorphisms, i.e., the manifolds are diffeomorphic. Moreover, as an application to the differentiable pinching problem, he proved in 32 that there exists a certain constant $\delta(n) \in(1 / 4,1)$ depending on a number $n$ such that if sectional curvature of a simply connected, compact Riemannian manifold $M$ of dimension $n$ is $\delta(n)$-pinched, then $M$ is diffeomorphic to the standard sphere. What astonishes us is that he defined such a distance between two manifolds for getting the differentiable sphere theorem more than ten years before Gromov's Hausdorff distance in [16].

\subsection{Applications of Theorem 1.3: Three differentiable sphere theorems}

Now, we are going to state applications of Theorem 1.3: Taking Theorem 1.1]into account, we consider the following setting. Let $M_{i}(i=1,2)$ be a compact manifold of dimension $n$ admitting a point $p_{i} \in M_{i}$ with a single cut point $q_{i} \in M_{i}$, and let $d_{M_{i}}\left(p_{i}, q_{i}\right)=\ell_{i}$, where $d_{M_{i}}$ denotes the distance function of $M_{i}$. Note that $M_{i}$ is homeomorphic to $S^{n}$. Choose a linear isometry $I: T_{p_{1}} M_{1} \longrightarrow T_{p_{2}} M_{2}$, where $T_{p_{i}} M_{i}$ denotes the tangent space of $M_{i}$ at $p_{i}$. For each $i=1,2$, let $\sigma_{q_{i}}^{p_{i}}$ be the diffeomorphism from $\mathbb{S}_{p_{i}}^{n-1}:=\left\{v \in T_{p_{i}} M_{i} \mid\|v\|=1\right\}$ onto $\mathbb{S}_{q_{i}}^{n-1}$ defined by

$$
\sigma_{q_{i}}^{p_{i}}\left(u_{i}\right):=-\dot{\tau}_{u_{i}}\left(\ell_{i}\right)
$$

where $\tau_{u_{i}}(t):=\exp _{p_{i}} t u_{i}$ for all $u_{i} \in \mathbb{S}_{p_{i}}^{n-1}$ and all $t \in\left[0, \ell_{i}\right]$. Thus, we have the diffeomorphism

$$
\sigma: \mathbb{S}_{q_{1}}^{n-1} \longrightarrow \mathbb{S}_{q_{2}}^{n-1}
$$

defined by $\sigma:=\sigma_{q_{2}}^{p_{2}} \circ I \circ \sigma_{p_{1}}^{q_{1}}$, where $\sigma_{p_{1}}^{q_{1}}:=\left(\sigma_{q_{1}}^{p_{1}}\right)^{-1}$. The bi-Lipschitz constant Lip ${ }^{\mathrm{b}}(\sigma)$ of $\sigma$ is given by

$$
\operatorname{Lip}^{\mathrm{b}}(\sigma):=\inf \left\{\ell \mid \ell^{-1}\|u-v\| \leq\|\sigma(u)-\sigma(v)\| \leq \ell\|u-v\| \text { for all } u, v \in \mathbb{S}_{q_{1}}^{n-1}\right\} .
$$

For a geodesic segment $\gamma:[0, \pi] \longrightarrow \mathbb{S}_{q_{1}}^{n-1}$ with $\|\dot{\gamma}\|:=\|d \gamma / d t\| \equiv 1$, let $c:[0, \pi] \longrightarrow \mathbb{S}_{q_{2}}^{n-1}$ be the curve defined by

$$
c:=\sigma \circ \gamma
$$

Then, we find that the relation between the differentiable structures of $M_{1}$ and $M_{2}$ depends on the map $\sigma$ above, i.e., the first application of Theorem 1.3 is stated as follows:

Differentiable Exotic Sphere Theorem I. If $\operatorname{Lip}^{\mathrm{b}}(\sigma)$ satisfies

$$
\|\ddot{c}\|^{2}-2 \operatorname{Lip}^{\mathrm{b}}(\sigma)^{-2} \leq 2\left\{\frac{\sqrt{2}-1}{2\left(e^{\pi}-1\right)}\right\}^{2}-1
$$


for all geodesic segments $\gamma([0, \pi]) \subset \mathbb{S}_{q_{1}}^{n-1}$ with $\|\dot{\gamma}\| \equiv 1$ where $c:=\sigma \circ \gamma$ and $\|\ddot{c}\|:=$ $\left\|d^{2} c / d t^{2}\right\|$, or if the $\sigma$ and $n$ satisfy

$$
\operatorname{Lip}^{\mathrm{b}}(\sigma)^{2} \leq 1+\left\{\frac{8}{\pi}(n-1)\right\}^{-\frac{1}{2}}
$$

then $M_{1}$ and $M_{2}$ are diffeomorphic.

Remark 1.12 We give two remarks on the differentiable exotic sphere theorem I and the outlines of the proof of it in order to show Theorem 1.3 to be useful:

- The assumption (1.6) does not depend on the dimension. Moreover, it implies that $\sigma$ is almost an isometry, and that $c$ is almost a geodesic segment on $\mathbb{S}_{q_{2}}^{n-1}$. The inevitability of the restriction of $\ddot{c}$ is discussed in Sect.3.4.

- The right side of (1.7) is the same constant as Karcher [21] estimated to get a sharper version of Shikata's theorem in [31].

- The outline of the proof in the case where (1.6) holds: Let $d_{M_{i}}\left(p_{i}, q_{i}\right):=\pi(i=1,2)$ by normalizing the metric. For each $\left(t, u_{1}\right) \in[0, \pi] \times \mathbb{S}_{p_{1}}^{n-1}$, we define the bi-Lipschitz homeomorphism $F: M_{1} \longrightarrow M_{2}$ by

$$
F\left(\exp _{p_{1}} t u_{1}\right):=\exp _{p_{2}}\left(t I\left(u_{1}\right)\right) \text {. }
$$

Note that $F$ is a diffeomorphism between $M_{1} \backslash\left\{q_{1}\right\}$ and $M_{2} \backslash\left\{q_{2}\right\}$. Thanks to (1.6), we see that $q_{1}$ is a non-singular point of $F$, and hence $F$ has no singular points on $M_{1}$. By Theorem [1.3, for a sufficiently small $\eta>0$, we can approximate $F$ via smooth immersions $f_{\eta}: M_{1} \longrightarrow M_{2}$. By a topological argument, we see that the local diffeomorphism $f_{\eta}$ is a bijection. Therefore, $M_{1}$ and $M_{2}$ are diffeomorphic.

- The outline of the proof in the case where (1.7) holds: Let $F: M_{1} \longrightarrow M_{2}$ denote the map defined by (1.8). And we embed $M_{2}$ into $\mathbb{R}^{m}$ isometrically, where $m \geq n+1$. By (1.7) and [21, Theorem 5.1], we can find $\delta>0$ such that a locally smooth approximation $\left.F_{\varepsilon}^{\left(q_{1}\right)}\right|_{B_{\delta}\left(q_{1}\right)}$ of $F$ is an immersion into $\mathbb{R}^{m}$ for all $\varepsilon \in(0, \delta)$, where $B_{\delta}\left(q_{1}\right):=\left\{x \in M_{1} \mid d_{M_{1}}\left(q_{1}, x\right)<\delta\right\}$. Then, we have the local diffeomorphism $F_{\varepsilon}: M_{1} \longrightarrow \mathbb{R}^{m}$ defined by $F_{\varepsilon}:=(1-\varphi) F+\varphi F_{\varepsilon}^{\left(q_{1}\right)}$. Here, $\varphi$ denotes a smooth function on $M_{1}$ satisfying $0 \leq \varphi \leq 1$ on $M_{1}, \varphi \equiv 1$ on $\overline{B_{r}\left(q_{1}\right)}$, and $\operatorname{supp} \varphi \subset B_{R}\left(q_{1}\right)$, where $0<r<R<\delta$. Choose a sufficiently small open neighborhood $U$ of $M_{2}$ in $\mathbb{R}^{m}$ so that the smooth (locally) distance projection $\pi: U \longrightarrow M_{2}$ is well-defined. Since $F_{\varepsilon}\left(M_{1}\right) \subset U$ for any sufficiently small $\varepsilon>0$, the map $f_{\varepsilon}:=\pi \circ F_{\varepsilon}: M_{1} \longrightarrow M_{2}$ is a local diffeomorphism. Since $f_{\varepsilon}$ is bijective, $M_{1}$ and $M_{2}$ are diffeomorphic. The fundamental course in the proof of Theorem 1.3 has a similar construction of $f_{\varepsilon}$, where we use the partition of unity.

Moreover, let $\bar{c}:[0, \pi] \longrightarrow T_{q_{2}} M_{2}$ be the smooth curve defined by

$$
\bar{c}(t):=c(0) \cos t+\dot{c}(0) \sin t,
$$

where $c$ is the curve in $\mathbb{S}_{q_{2}}^{n-1}$ defined by (1.5) and we set $\dot{c}(t):=d c / d t$. Note that $\|\dot{c}(0)\| \neq 1$ is possible. Then, we can replace (1.6) with the following condition (1.10): 
Differentiable Exotic Sphere Theorem II. If $\sigma$ satisfies

$$
\angle(\bar{c}(t), c(t))<\frac{\pi}{2}
$$

for all geodesic segments $\gamma([0, \pi]) \subset \mathbb{S}_{q_{1}}^{n-1}$ with $\|\dot{\gamma}\| \equiv 1$, then $M_{1}$ and $M_{2}$ are diffeomorphic.

Remark 1.13 We find (1.10) in the process for proving lemmas that we need for the proof of the differentiable exotic sphere theorem I in the case where (1.6) holds. By (1.10), $q_{1}$ is non-singular for the map $F$ defined by (1.8), and hence $F$ has no singular points on $M_{1}$. By Theorem 1.3, we get the assertion.

Next, we will state the third application of Theorem 1.3 and its corollaries: Let $M$ be a compact Riemannian manifold of dimension $n$. For any two distinct points $p, q \in M$, we set

$$
D(p):=\{x \in M \mid d(p, x)<d(q, x)\}, \quad D(q):=\{x \in M \mid d(p, x)>d(q, x)\}
$$

and

$$
E_{p, q}:=\{x \in M \mid d(p, x)=d(q, x)\},
$$

where $d$ denotes the distance function of $M$. Moreover, for a point $x \in M$, we set

$$
\operatorname{Crit}(x):=\left\{y \in M \mid o \in \partial d_{x}(y)\right\}
$$

With these notations, we have

Differentiable Twisted Sphere Theorem. Take any two distinct points $p, q \in M$. If

$$
D(p) \cap \operatorname{Crit}(p)=\{p\}, \quad D(q) \cap \operatorname{Crit}(q)=\{q\},
$$

and if for any geodesic segments $\alpha$ and $\beta$ emanating from each point $x \in E_{p, q}$ to $p$ and $q$, respectively,

$$
\angle(\dot{\alpha}(0), \dot{\beta}(0))>\frac{\pi}{2}
$$

holds at $x$, then

(T-1) $M$ is a twisted sphere, and

(T-2) there exists a bi-Lipschitz homeomorphism between $M$ and $S^{n}(1)$ which is a diffeomorphism except for the point $q$, where $S^{n}(1):=\left\{v \in \mathbb{R}^{n+1} \mid\|v\|=1\right\}$.

Furthermore, we have that

(T-3) there exists a diffeomorphism $\sigma_{q}^{p}: \mathbb{S}_{p}^{n-1} \longrightarrow \mathbb{S}_{q}^{n-1}$ defined similarly to (1.2) such that if the following condition (a) or (b) is satisfied, then $M$ and $S^{n}(1)$ are diffeomorphic: 
(a) $\operatorname{Lip}^{\mathrm{b}}\left(\left(\sigma_{q}^{p}\right)^{-1}\right)$ satisfies (1.6) for all geodesic segments $\gamma([0, \pi]) \subset \mathbb{S}_{q}^{n-1}$ with $\|\dot{\gamma}\| \equiv 1$, where the curve $c:[0, \pi] \longrightarrow \mathbb{S}_{S}^{n-1}$ is given by $c:=\sigma_{S}^{N} \circ I \circ\left(\sigma_{q}^{p}\right)^{-1} \circ \gamma$, and we put $N:=(0,0, \ldots, 1), S:=(0,0, \ldots,-1) \in S^{n}(1)$, or if $\left(\sigma_{q}^{p}\right)^{-1}$ and $n$ satisfy (1.7);

(b) $\left(\sigma_{q}^{p}\right)^{-1}$ satisfies (1.10).

Note that the curve $\bar{c}:[0, \pi] \longrightarrow T_{S} S^{n}(1)$ in (1.10) is defined by (1.9) for the $c=$ $\sigma_{S}^{N} \circ I \circ\left(\sigma_{q}^{p}\right)^{-1} \circ \gamma$.

Remark 1.14 We give two remarks on the differentiable twisted sphere theorem and several related topics for it:

- Since $M$ is twisted by (T-1), $M$ admits a metric such that the cut locus of some point in $M$ is a single point by Weinstein's deformation technique for metrics. See Remark 4.3 for details.

- The diffeomorphism $\sigma_{q}^{p}$ in (T-3) induces a boundary diffeomorphism $h_{\sigma_{q}^{p}}: S^{n-1} \longrightarrow$ $S^{n-1}$ such that $M=D^{n} \cup_{\sigma_{q}^{p}} D^{n}$, where $D^{n}$ denotes the standard $n$-disc and $S^{n-1}=$ $\partial D^{n}$ (See Sect.4). Thus, by taking the contrapositive of (T-3), we could define clearly a boundary diffeomorphism to get an exotic sphere $\Sigma^{n}(n>4)$ from a twisted one. The fact that there are few explicit examples of such maps is worthy of note. E.g., Durán's boundary diffeomorphism for an $\Sigma^{7}$ in [10].

- Donaldson and Sullivan [9] proved that there are smooth 4-manifolds which are homeomorphic, but not bi-Lipschitz.

- Let $M$ be a piecewise linear (PL) $n$-manifold 2 of dimension $n \geq 5$ which has the homotopy type of $S^{n}$. Then, the Stallings-Zeeman theorem ([36] together with [40]) says that $M \backslash\{$ point $\}$ is PL-homeomorphic to $\mathbb{R}^{n}$.

- For any closed Riemannian manifold $M$ of dimension $n \geq 2$, Cheeger and Colding [4] found a positive number $\delta(n)$ depending on $n$ such that if $\operatorname{Ric}_{M} \geq n-1$ and $\operatorname{vol}(M)>\operatorname{vol}\left(S^{n}(1)\right)-\delta(n)$, then $M$ is diffeomorphic to $S^{n}(1)$, where $\operatorname{Ric}_{M}$ denotes Ricci curvature of $M$, and $\operatorname{vol}(M)$ denotes the volume of $M$. Note that their result is a generalization of the pioneering work of Otsu-Shiohama-Yamaguchi [27] in the sectional curvature case.

Next, we will state three corollaries of the differentiable twisted sphere theorem: Applying the same argument in the proof of (T-2) to any twisted sphere, we have

Corollary 1.15 For any twisted sphere $\Sigma^{n}$ of general dimension $n$, there exists a biLipschitz homeomorphism between $\Sigma^{n}$ and $S^{n}(1)$ which is a diffeomorphism except for a single point. In particular, if $n>4$, then there exists such a map between each exotic sphere $\Sigma^{n}$ and $S^{n}(1)$.

\footnotetext{
${ }^{2} \mathrm{~A}$ PL $n$-manifold is, by definition, a polyhedron admitting a linear triangulation that satisfies that the link of each vertex is combinatorially equivalent to the boundary of the $n$-simplex.
} 
Remark 1.16 Let $D^{n}$ be the standard $n$-disc and $S^{n-1}=\partial D^{n}$. Combining with results of Munkres [26], Kervaire-Milnor [22, and Cerf [3] $]^{3}$, we see that if $n \leq 6$, then the group $\Gamma_{n}:=\operatorname{Diff}\left(S^{n-1}\right) / \operatorname{Diff}\left(D^{n}\right)$ is trivial. Here, $\operatorname{Diff}(X)$ denotes the topological group of orientation preserving diffeomorphisms of a smooth manifold $X$. Hence, every twisted $n$-sphere of dimension $n \leq 6$ is diffeomorphic to $S^{n}(1)$.

Since $\Gamma_{4}=0$ as in the above, there is no twisted sphere but the standard sphere. Hence, if an exotic 4 -sphere $\Sigma^{4}$ exists, then $\Sigma^{4}$ is not twisted. Thus, we have

Corollary 1.17 If $\Sigma^{4}$ exists, then $\Sigma^{4}$ does not satisfy (1.11), or (1.12).

Remark 1.18 So far many topological 4-spheres regarded as exotic are indeed standard. E.g., Akbulut's [1] and Gompf's [13]. We refer to [12] about the difficulty to solve SPC4. We can find an interesting work on the relationship between Stein 4-manifolds and SPC4 in Yasui's 39.

Let $M$ be a Grove-Shiohama type $n$-sphere, and let $p, q \in M$ such that $d(p, q)=$ $\operatorname{diam}(M)$, where $d$ denotes the distance function of $M$. Then, Toponogov's comparison theorem shows the following process: First $M \backslash B_{\pi / 2}(p)$ is convex, where $B_{\pi / 2}(p):=\{x \in$ $M \mid d(p, x)<\pi / 2\}$, second $q$ is a unique point, and finally $\operatorname{Crit}(p)=\{p, q\}$ (See [18], or [23], 24 for details). By the same process above, we have $\operatorname{Crit}(q)=\{p, q\}$ since $K_{M} \geq 1$ everywhere. Thus, $M$ satisfies (1.11) and (1.12). By the differentiable twisted sphere theorem, we have

Corollary 1.19 Let $M$ be a Grove-Shiohama type $n$-sphere, and let $p, q \in M$ such that $d(p, q)=\operatorname{diam}(M)$. Then, there exists a bi-Lipschitz homeomorphism between $M$ and $S^{n}(1)$ which is a diffeomorphism except for $q$.

Remark 1.20 We give a remark on Corollary 1.19 and two related results for it:

- Since any Grove-Shiohama type $n$-sphere $M$ is twisted, $M$ of dimension $n \leq 6$ is diffeomorphic to $S^{n}(1)$. See Remark 1.16 above.

- Grove and Wilhelm [19] proved that every Grove-Shiohama type $n$-sphere is diffeomorphic to $S^{n}(1)$ if the diffeomorphism stability question is "yes" in their sense.

- Petersen and Wilhelm [28] announced that the Gromoll-Meyer exotic sphere in [14] admits a metric with positive sectional curvature everywhere.

The organization of this article is as follows: Sect.2 has three subsections. In Sect.2.1, we study an approximation of a Lipschitz function on a Riemannian manifold $M$. Using non-smooth analysis, we prove, as a main lemma (Lemma 2.14), that (1.12) also holds for two gradient vector fields on a compact set in $M$ of smooth approximations of two distance functions from distinct points in $M$. In Sect.2.2, we establish an approximation method for a Lipschitz map from a compact Riemannian manifold into a Riemannian manifold using some techniques from non-smooth analysis and the partition of unity, and prove

\footnotetext{
${ }^{3}$ An alternative proof of $\Gamma_{4}=0$ is found in Eliashberg's [11.
} 
our main theorem, Theorem 1.3, applying the method. In Sect.2.3, we prove Corollary 1.10 applying Theorem 1.3. Sect. 3 has four subsections. In the first two subsections 3.1 and 3.2, we give preliminaries to proofs of the differentiable exotic sphere theorems I and II in assuming each of the conditions, i.e., (1.6), (1.7), and (1.10). In Sect.3.3, we prove the two theorems. In Sect.3.4, we discuss why we need the restriction of $\ddot{c}$ in (1.6) for the theorem I. In Sect.4, we prove the differentiable twisted sphere theorem applying Lemma 2.14 and Theorem 1.3 .

\section{Approximations of Lipschitz maps via immersions}

We prove here our main theorem, Theorem 1.3. For the proof, we establish an approximation method for a Lipschitz map from a compact Riemannian manifold into a Riemannian manifold using non-smooth analysis. As was mentioned in Remark 1.4, this approximation method generalizes the whole method of Grove-Shiohama [18. Note that we do not assume curvature assumptions at all, and that the smoothing technique bases on the partition of unity, while those in [18], [21] depend on the center of mass technique constructed by Grove and Karcher [17].

\subsection{Approximations of Lipschitz functions}

We first treat an approximation of a Lipschitz function $f$ on a Riemannian manifold $M$ of dimension $n$. As a main lemma (Lemma 2.14) in this subsection, we prove that our approximation method keeps (1.12) for two gradient vector fields $\nabla\left(d_{p}\right)_{\varepsilon}, \nabla\left(d_{q}\right)_{\varepsilon}$ on a compact set in $M$ of smooth approximations $\left(d_{p}\right)_{\varepsilon},\left(d_{q}\right)_{\varepsilon}$ of two distance functions $d_{p}, d_{q}$ from distinct points $p, q \in M$, where $d$ denotes the distance function of $M$. This lemma will be applied to the proof of the differentiable twisted sphere theorem in Sect.4.

Take any point $p \in M$, and fix it. Since the exponential map $\exp _{p}$ on $T_{p} M$ is a diffeomorphism from $\mathbb{B}_{r}\left(o_{p}\right)$ onto $B_{r}(p)$ for a sufficiently small $r>0$, we denote by $\exp _{p}^{-1}$ the inverse map of $\left.\exp _{p}\right|_{\mathbb{B}_{r}\left(o_{p}\right)}$. Here we set

$$
\mathbb{B}_{r}\left(o_{p}\right):=\left\{v \in T_{p} M \mid\|v\|<r\right\}, \quad B_{r}(p):=\{q \in M \mid d(p, q)<r\},
$$

where $o_{p}$ denotes the origin of $T_{p} M$. In what follows, we identify $T_{p} M$ with Euclidean $n$-dimensional space $\left(\mathbb{R}^{n},\langle\cdot, \cdot\rangle\right)$. Then, we may define a smooth approximation of the $f$ around $p$ :

Definition 2.1 For each $\varepsilon>0$, let $f_{\varepsilon}^{(p)}: B_{r}(p) \longrightarrow \mathbb{R}$ denote the function defined by

$$
f_{\varepsilon}^{(p)}(q):=\int_{\mathbb{R}^{n}} f(q(y)) \rho_{\varepsilon}(y) d y=\int_{\mathbb{R}^{n}} f\left(\exp _{p}(y)\right) \rho_{\varepsilon}\left(\exp _{p}^{-1} q-y\right) d y
$$

where we set $q(y):=\exp _{p}\left(\exp _{p}^{-1} q-y\right)$, and the function $\rho_{\varepsilon}$ denotes the mollifier. Refer to [31], 21], or [18] for details of the mollifier.

Choose a locally finite covering $\left\{B_{r_{i}}\left(p_{i}\right)\right\}$ of strongly convex balls of $M$ such that

$$
M=\cup_{i} B_{r_{i} / 2}\left(p_{i}\right) .
$$


Here, a subset $C \subset M$ is said to be strongly convex if for any two points $x, y$ in the closure $\bar{C}$ of $C$, there exists a unique minimal geodesic segment $\gamma:[0,1] \longrightarrow M$ emanating from $x=\gamma(0)$ to $y=\gamma(1)$ such that $\gamma((0,1)) \subset C$. Note that for each $x \in M$, there exists a strongly convex ball of its center $x$ (Whitehead's convexity theorem [38]).

Let $\left\{\psi_{i}\right\}_{i=1}^{\infty}$ be the partition of unity subordinate to $\left\{B_{r_{i} / 2}\left(p_{i}\right)\right\}$. Define the smooth approximation $f_{\varepsilon}$ of the $f$ on $M$ by

$$
f_{\varepsilon}(q):=\sum_{i=1}^{\infty} \psi_{i}(q) f_{\varepsilon}^{\left(p_{i}\right)}(q) .
$$

By definition,

$$
f_{\varepsilon}^{\left(p_{i}\right)}(q)=\int_{\mathbb{R}^{n}} f\left(q_{i}(y)\right) \rho_{\varepsilon}(y) d y
$$

for $q \in B_{r_{i}}\left(p_{i}\right)$, where

$$
q_{i}(y):=\exp _{p_{i}}\left(\exp _{p_{i}}^{-1} q-y\right) .
$$

Lemma 2.2 For each center $p_{i}$ of $B_{r_{i}}\left(p_{i}\right)$,

$$
\sup _{q \in \operatorname{supp} \psi_{i}}\left|f_{\varepsilon}^{\left(p_{i}\right)}(q)-f(q)\right| \leq \varepsilon \cdot \operatorname{Lip}(f) \cdot \operatorname{Lip}\left(\exp _{p_{i}} \mid \mathbb{B}_{r_{i}}\left(o_{p_{i}}\right)\right)
$$

holds for all $\varepsilon \in\left(0, \varepsilon_{i}\right)$, where $\varepsilon_{i}:=r_{i}-\max \left\{\left\|\exp _{p_{i}}^{-1} q\right\| \mid q \in \operatorname{supp} \psi_{i}\right\}$, and $\operatorname{Lip}(f)$ and $\operatorname{Lip}\left(\left.\exp _{p_{i}}\right|_{\mathbb{B}_{r_{i}}\left(o_{p_{i}}\right)}\right)$ denote the Lipschitz constants of $f$ and $\left.\exp _{p}\right|_{\mathbb{B}_{r_{i}}\left(o_{p_{i}}\right)}$, respectively, i.e.,

$$
\operatorname{Lip}(f):=\sup \left\{\frac{\left|f\left(q_{1}\right)-f\left(q_{2}\right)\right|}{d\left(q_{1}, q_{2}\right)} \mid q_{1}, q_{2} \in M, q_{1} \neq q_{2}\right\}
$$

and

$$
\operatorname{Lip}\left(\left.\exp _{p_{i}}\right|_{\mathbb{B}_{r_{i}}\left(o_{p_{i}}\right)}\right):=\sup \left\{\frac{d\left(\exp _{p_{i}} v, \exp _{p_{i}} w\right)}{\|v-w\|} \mid v, w \in \mathbb{B}_{r_{i}}\left(o_{p_{i}}\right), v \neq w\right\} .
$$

Proof. Take any point $q \in \operatorname{supp} \psi_{i}$. Then, for any $y \in \mathbb{B}_{\varepsilon_{i}}\left(o_{p_{i}}\right)$, we have, by the triangle inequality,

$$
\left\|\exp _{p_{i}}^{-1} q-y\right\| \leq\left\|\exp _{p_{i}}^{-1} q\right\|+\|y\|<r_{i} .
$$

Thus, $\exp _{p_{i}}^{-1} q-y$ and $\exp _{p_{i}}^{-1} q$ are elements of $\mathbb{B}_{r_{i}}\left(o_{p_{i}}\right)$ for all $y \in \mathbb{B}_{\varepsilon_{i}}\left(o_{p_{i}}\right)$. Hence,

$$
d\left(q_{i}(y), q\right) \leq \operatorname{Lip}\left(\exp _{p_{i}} \mid \mathbb{B}_{r_{i}}\left(o_{p_{i}}\right)\right)\left\|\left(\exp _{p_{i}}^{-1} q-y\right)-\exp _{p_{i}}^{-1} q\right\|=\operatorname{Lip}\left(\exp _{p_{i}} \mid \mathbb{B}_{r_{i}}\left(o_{p_{i}}\right)\right)\|y\| .
$$

On the other hand, since $\int_{\mathbb{R}^{n}} \rho_{\varepsilon}(y) d y=1$, we have $f(q)=\int_{\|y\|<\varepsilon} f(q) \rho_{\varepsilon}(y) d y$. Then,

$$
\begin{aligned}
\left|f_{\varepsilon}^{\left(p_{i}\right)}(q)-f(q)\right| & \leq \int_{\|y\|<\varepsilon}\left|f\left(q_{i}(y)\right)-f(q)\right| \rho_{\varepsilon}(y) d y \\
& \leq \operatorname{Lip}(f) \int_{\|y\|<\varepsilon} d\left(q_{i}(y), q\right) \rho_{\varepsilon}(y) d y
\end{aligned}
$$

where note that $\|y\|<\varepsilon$ when $\rho_{\varepsilon}(y) \neq 0$. Combining (2.5) with (2.6), we get (2.3) . 
Take any $p \in M$ and any $p_{i}$ with $p \in \operatorname{supp} \psi_{i}$, and fix them in the following. Moreover, we assume $q \in \operatorname{supp} \psi_{i}$ in this situation.

Lemma 2.3 For any $\tilde{u} \in \mathbb{S}_{q}^{n-1}:=\left\{v \in T_{q} M \mid\|v\|=1\right\}$

$$
\left(d f_{\varepsilon}^{\left(p_{i}\right)}\right)_{q}(\tilde{u})=\int_{\mathbb{R}^{n}} d f_{q_{i}(y)}\left(Y_{y}^{(\tilde{u})}(1)\right) \rho_{\varepsilon}(y) d y
$$

holds, where $q_{i}(y) \in M$ is by definition (2.2), and

$$
Y_{y}^{(\tilde{u})}(t):=\left.\frac{\partial}{\partial s} \exp _{p_{i}} t\left(\exp _{p_{i}}^{-1}\left(\exp _{q} s \tilde{u}\right)-y\right)\right|_{s=0}
$$

is a Jacobi field along the geodesic $\exp _{p_{i}} t\left(\exp _{p_{i}}^{-1} q-y\right)$ for each $y$.

Proof. Since

$$
\left(d f_{\varepsilon}^{\left(p_{i}\right)}\right)_{q}(\tilde{u})=\left.\frac{d}{d s} f_{\varepsilon}^{\left(p_{i}\right)}\left(\exp _{q} s \tilde{u}\right)\right|_{s=0},
$$

we get (2.7) by (2.1).

For a pair of points $q_{1}$ and $q_{2}$ of $M$ admitting a unique minimal geodesic segment $\gamma$, let $\tau_{q_{2}}^{q_{1}}: T_{q_{1}} M \longrightarrow T_{q_{2}} M$ denote the parallel transportation along $\gamma$. In what follows, we will omit brackets of $\tau_{q_{2}}^{q_{1}}(u)\left(u \in T_{q_{1}} M\right)$ for simplicity, i.e, $\tau_{q_{2}}^{q_{1}} u:=\tau_{q_{2}}^{q_{1}}(u)$.

Lemma 2.4 Let $\widetilde{U}_{q_{i}(y)}:=\tau_{q_{i}(y)}^{q} \tilde{u}$. Then, we have

$$
\left|\left(d f_{\varepsilon}^{\left(p_{i}\right)}\right)_{q}(\tilde{u})-\int_{\mathbb{R}^{n}} d f_{q_{i}(y)}\left(\widetilde{U}_{q_{i}(y)}\right) \rho_{\varepsilon}(y) d y\right| \leq \operatorname{Lip}(f) \sup _{y \in \mathbb{B}_{\varepsilon}\left(o_{p_{i}}\right)}\left\|Y_{y}^{(\tilde{u})}(1)-\widetilde{U}_{q_{i}(y)}\right\| .
$$

Proof. By Lemma 2.3 and (2.4), we get

$$
\begin{aligned}
\left|\left(d f_{\varepsilon}^{\left(p_{i}\right)}\right)_{q}(\tilde{u})-\int_{\mathbb{R}^{n}} d f_{q_{i}(y)}\left(\widetilde{U}_{q_{i}(y)}\right) \rho_{\varepsilon}(y) d y\right| & \leq \int_{\mathbb{R}^{n}}\left|d f_{q_{i}(y)}\left(Y_{y}^{(\tilde{u})}(1)-\widetilde{U}_{q_{i}(y)}\right)\right| \rho_{\varepsilon}(y) d y \\
& \leq \operatorname{Lip}(f) \sup _{y \in \mathbb{B}_{\varepsilon}\left(o_{p_{i}}\right)}\left\|Y_{y}^{(\tilde{u})}(1)-\widetilde{U}_{q_{i}(y)}\right\| .
\end{aligned}
$$

Lemma 2.5 For any $\eta>0$, there exists a number $\varepsilon_{i}(\eta)>0$ such that

$$
\sup \left\{\left\|Y_{y}^{(\tilde{u})}(1)-\widetilde{U}_{q_{i}(y)}\right\| \mid y \in \mathbb{B}_{\varepsilon_{i}(\eta)}\left(o_{p_{i}}\right), q \in \operatorname{supp} \psi_{i}, \tilde{u} \in \mathbb{S}_{q}^{n-1}\right\}<\eta .
$$

Proof. Let $\eta>0$ be an arbitrary number. Take any $q \in \operatorname{supp} \psi_{i}$ and any $\tilde{u} \in \mathbb{S}_{q}^{n-1}$. Since $Y_{y}^{(\tilde{u})}(1)=\widetilde{U}_{q_{i}(y)}=\tilde{u}$ for $y=o$, there exists a positive number $\varepsilon\left(q, \tilde{u}, p_{i}, \eta\right)>0$ such that $\left\|Y_{y}^{(\tilde{u})}(1)-\widetilde{U}_{q_{i}(y)}\right\|<\eta$ for all $y \in \mathbb{B}_{\varepsilon\left(q, \tilde{u}, p_{i}, \eta\right)}\left(o_{p_{i}}\right)$. Since $\operatorname{supp} \psi_{i}$ and $\mathbb{S}_{q}^{n-1}$ are compact, there exists a positive number $\varepsilon_{i}(\eta)$ such that $\left\|Y_{y}^{(\tilde{u})}(1)-\widetilde{U}_{q_{i}(y)}\right\|<\eta$ for all $q \in \operatorname{supp} \psi_{i}$, $\tilde{u} \in \mathbb{S}_{q}^{n-1}$, and $y \in \mathbb{B}_{\varepsilon_{i}(\eta)}\left(o_{p_{i}}\right)$. 
For each $v \in \mathbb{R}^{n}=T_{p} M$, let $\pi_{p}(v)$ be the nearest point on $\partial f(p)$ from $v$. Since $\partial f(p)$ is convex, the nearest point is uniquely determined. Moreover, it is easy to check that the map $\pi_{p}: \mathbb{R}^{n} \longrightarrow \partial f(p)$ is continuous.

\section{Lemma 2.6 Set}

$$
v_{\varepsilon}^{(i)}:=\int_{\mathbb{R}^{n}} \pi_{p}\left(\tau_{p}^{q} \tau_{q}^{q_{i}(y)} \nabla f\left(q_{i}(y)\right)\right) \rho_{\varepsilon}(y) d y
$$

and $u:=\tau_{p}^{q} \tilde{u}$. Then, we have

$$
\left|\int_{\mathbb{R}^{n}} d f_{q_{i}(y)}\left(\widetilde{U}_{q_{i}(y)}\right) \rho_{\varepsilon}(y) d y-\left\langle v_{\varepsilon}^{(i)}, u\right\rangle\right| \leq \int_{\mathbb{R}^{n}}\left\|\left(1-\pi_{p}\right)\left(\tau_{p}^{q} \tau_{q}^{q_{i}(y)} \nabla f\left(q_{i}(y)\right)\right)\right\| \rho_{\varepsilon}(y) d y
$$

where we set $\widetilde{U}_{q_{i}(y)}:=\tau_{q_{i}(y)}^{q} \tilde{u}$.

Proof. It is easy to check that $d f_{q_{i}(y)}\left(\widetilde{U}_{q_{i}(y)}\right)=\left\langle\tau_{p}^{q} \tau_{q}^{q_{i}(y)} \nabla f\left(q_{i}(y)\right), u\right\rangle$. Thus, by the Cauchy-Schwarz inequality, we get

$$
\begin{aligned}
\left|\int_{\mathbb{R}^{n}} d f_{q_{i}(y)}\left(\widetilde{U}_{q_{i}(y)}\right) \rho_{\varepsilon}(y) d y-\left\langle v_{\varepsilon}^{(i)}, u\right\rangle\right| & =\left|\int_{\mathbb{R}^{n}}\left\langle\left(1-\pi_{p}\right)\left(\tau_{p}^{q} \tau_{q}^{q_{i}(y)} \nabla f\left(q_{i}(y)\right)\right), u\right\rangle \rho_{\varepsilon}(y) d y\right| \\
& \leq \int_{\mathbb{R}^{n}}\left\|\left(1-\pi_{p}\right)\left(\tau_{p}^{q} \tau_{q}^{q_{i}(y)} \nabla f\left(q_{i}(y)\right)\right)\right\| \rho_{\varepsilon}(y) d y
\end{aligned}
$$

Definition 2.7 A map $F: \mathbb{R}^{n} \longrightarrow \mathbb{R}^{k}$ is called a locally $L^{1}$-map if each $F^{i}: \mathbb{R}^{n} \longrightarrow \mathbb{R}$ is a locally $L^{1}$-function, where $F^{i}(x)$ denotes the $i$-th component of $F(x) \in \mathbb{R}^{k}$.

Lemma 2.8 Let $F: \mathbb{R}^{n} \longrightarrow \mathbb{R}^{k}$ be a locally $L^{1}-$ map, and $C \subset \mathbb{R}^{k}$ a compact convex set. If $F\left(\mathbb{R}^{n}\right) \subset C$, then $\int_{\mathbb{R}^{n}} \rho(x) F(x) d x \in C$ for all non-negative continuous function $\rho: \mathbb{R}^{n} \longrightarrow \mathbb{R}$ whose support is compact with $\int_{\mathbb{R}^{n}} \rho(x) d x=1$.

Proof. Let $\left\{H_{\alpha}\right\}_{\alpha \in \Gamma}$ be the family of all closed half spaces in $\mathbb{R}^{k}$. It is well known that $K=\bigcap_{K \subset H_{\alpha}} H_{\alpha}$ for any closed convex subset $K$ of $\mathbb{R}^{k}$. Choose any closed half space $H_{\alpha}$ with $C \subset H_{\alpha}$. Let $n_{\alpha}$ denote the inward pointing unit normal vector of $H_{\alpha}$ and $v_{\alpha} \in \mathbb{R}^{k}$ a nearest point of $C$ from $\partial H_{\alpha}$. Then, $\left\langle F(x)-v_{\alpha}, n_{\alpha}\right\rangle \geq 0$ holds for all $x$, since $F(x) \in C$. Since $\int_{\mathbb{R}^{n}} \rho(x) d x=1, \int_{\mathbb{R}^{n}} \rho(x) v_{\alpha} d x=v_{\alpha}$. Thus,

$$
\left\langle\int \rho(x) F(x) d x-v_{\alpha}, n_{\alpha}\right\rangle=\int \rho(x)\left\langle F(x)-v_{\alpha}, n_{\alpha}\right\rangle d x \geq 0,
$$

and hence $\int_{\mathbb{R}^{n}} \rho(x) F(x) d x \in H_{\alpha}$ for all $H_{\alpha}$ with $C \subset H_{\alpha}$. Thus, $\int_{\mathbb{R}^{n}} \rho(x) F(x) d x \in C$.

Lemma 2.9 For any $\eta>0$, there exists a number $\delta(p, \eta)>0$ such that

$$
\tau_{p}^{q_{1}} \tau_{q_{1}}^{q_{2}} \nabla f\left(q_{2}\right) \in \partial f(p)_{\eta}:=\bigcup_{v \in \partial f(p)} \mathbb{B}_{\eta}(v)
$$

holds for all $q_{1} \in B_{\delta(p, \eta)}(p)$ and $q_{2} \in B_{\delta(p, \eta)}(p) \cap\left(M \backslash E_{f}\right)$. 
Proof. Take any two sequences $\left\{x_{i}\right\}$ of $x_{i} \in M$ and $\left\{y_{i}\right\}$ of $y_{i} \in M \backslash E_{f}$ both of which are convergent to the point $p$. If the limit $\lim _{i \rightarrow \infty} \nabla f\left(y_{i}\right)$ exists, then $\lim _{i \rightarrow \infty} \tau_{p}^{x_{i}} \tau_{x_{i}}^{y_{i}} \nabla f\left(y_{i}\right)=$ $\lim _{i \rightarrow \infty} \nabla f\left(y_{i}\right) \in \partial f(p)$. This implies the existence of the positive number $\delta(p, \eta)$.

Lemma 2.10 For any $\eta>0$, there exist numbers $\delta_{1}(p, \eta)>0$ and $\varepsilon\left(p, p_{i}, \eta\right)>0$ such that

$$
\tau_{p}^{q} \nabla f_{\varepsilon}^{\left(p_{i}\right)}(q) \in \partial f(p)_{\eta}
$$

for all $q \in B_{\delta_{1}(p, \eta)}(p) \cap \operatorname{supp} \psi_{i}$, and for all $\varepsilon \in\left(0, \varepsilon\left(p, p_{i}, \eta\right)\right)$.

Proof. It follows from Lemmas 2.4 and 2.5 that

$$
\left|\left(d f_{\varepsilon}^{\left(p_{i}\right)}\right)_{q}(\tilde{u})-\int_{\mathbb{R}^{n}} d f_{q_{i}(y)}\left(\widetilde{U}_{q_{i}(y)}\right) \rho_{\varepsilon}(y) d y\right|<\frac{\eta}{2}
$$

for all $\varepsilon \in\left(0, \varepsilon_{i}(\eta / 2 \operatorname{Lip}(f))\right), q \in \operatorname{supp} \psi_{i}$, and $\tilde{u} \in \mathbb{S}_{q}^{n-1}$. If $\varepsilon$ is not greater than

$$
\min \left\{r_{i}-\max _{q \in \operatorname{supp} \psi_{i}}\left\|\exp _{p_{i}}^{-1} q\right\|, \quad \frac{\delta(p, \eta / 2)}{2 \operatorname{Lip}\left(\exp _{p_{i}} \mid \mathbb{B}_{r_{i}}\left(o_{p_{i}}\right)\right.}\right\}
$$

then $\left\|\exp _{p_{i}}^{-1} q-y\right\| \leq\left\|\exp _{p_{i}}^{-1} q\right\|+\|y\|<r_{i}$ for all $y \in \mathbb{B}_{\varepsilon}\left(o_{p_{i}}\right)$. Hence,

$$
d\left(q_{i}(y), q\right) \leq \operatorname{Lip}\left(\left.\exp _{p_{i}}\right|_{\mathbb{B}_{r_{i}}\left(o_{p_{i}}\right)}\right)\|y\|<\frac{\delta(p, \eta / 2)}{2}
$$

for all $y \in \mathbb{B}_{\varepsilon}\left(o_{p_{i}}\right)$. Thus, we have

$$
d\left(p, q_{i}(y)\right) \leq d(p, q)+d\left(q, q_{i}(y)\right)<\frac{\delta(p, \eta / 2)}{2}+\frac{\delta(p, \eta / 2)}{2}=\delta(p, \eta / 2)
$$

for all $q \in \operatorname{supp} \psi_{i} \cap B_{\delta_{1}(p, \eta)}(p)$, where we set $\delta_{1}(p, \eta):=\delta(p, \eta / 2) / 2$. Let

$$
\varepsilon\left(p, p_{i}, \eta\right):=\min \left\{\varepsilon_{i}(\eta / 2 \operatorname{Lip}(f)), \quad \frac{\delta_{1}(p, \eta)}{\operatorname{Lip}\left(\exp _{p_{i}} \mid \mathbb{B}_{r_{i}}\left(o_{p_{i}}\right)\right.}, \quad r_{i}-\max _{q \in \operatorname{supp} \psi_{i}}\left\|\exp _{p_{i}}^{-1} q\right\|\right\} .
$$

From Lemma 2.9, we get

$$
\left\|\left(1-\pi_{p}\right)\left(\tau_{p}^{q} \tau_{q}^{q_{i}(y)} \nabla f\left(q_{i}(y)\right)\right)\right\|<\frac{\eta}{2}
$$

for all $q \in B_{\delta_{1}(p, \eta)}(p)$ and almost all $y \in \mathbb{B}_{\varepsilon\left(p, p_{i}, \eta\right)}\left(o_{p_{i}}\right)$. Therefore, by Lemma 2.6, we obtain

$$
\left|\int_{\mathbb{R}^{n}} d f_{q_{i}(y)}\left(\widetilde{U}_{q_{i}(y)}\right) \rho_{\varepsilon}(y) d y-\left\langle v_{\varepsilon}^{(i)}, u\right\rangle\right|<\frac{\eta}{2}
$$

for all $q \in B_{\delta_{1}(p, \eta)}(p)$ and all $\varepsilon \in\left(0, \varepsilon\left(p, p_{i}, \eta\right)\right)$. By the triangle inequality and the equations (2.9), (2.10), we obtain

$$
\left|\left(d f_{\varepsilon}^{\left(p_{i}\right)}\right)_{q}(\tilde{u})-\left\langle v_{\varepsilon}^{(i)}, u\right\rangle\right|<\eta
$$


for all $\tilde{u} \in \mathbb{S}_{q}^{n-1}, q \in \operatorname{supp} \psi_{i} \cap B_{\delta_{1}(p, \eta)}(p)$, and $\varepsilon \in\left(0, \varepsilon\left(p, p_{i}, \eta\right)\right)$. Since $\left(d f_{\varepsilon}^{\left(p_{i}\right)}\right)_{q}(\tilde{u})=$ $\left\langle\tau_{p}^{q} \nabla f_{\varepsilon}^{\left(p_{i}\right)}(q), u\right\rangle$ and $\tilde{u}$ is arbitrarily chosen, we have, by (2.11),

$$
\left\|\tau_{p}^{q} \nabla f_{\varepsilon}^{\left(p_{i}\right)}(q)-v_{\varepsilon}^{(i)}\right\|=\frac{\left|\left\langle\tau_{p}^{q} \nabla f_{\varepsilon}^{\left(p_{i}\right)}(q)-v_{\varepsilon}^{(i)}, \tau_{p}^{q} \nabla f_{\varepsilon}^{\left(p_{i}\right)}(q)-v_{\varepsilon}^{(i)}\right\rangle\right|}{\left\|\tau_{p}^{q} \nabla f_{\varepsilon}^{\left(p_{i}\right)}(q)-v_{\varepsilon}^{(i)}\right\|}<\eta
$$

for all $q \in \operatorname{supp} \psi_{i} \cap B_{\delta_{1}(p, \eta)}(p)$ and $\varepsilon \in\left(0, \varepsilon\left(p, p_{i}, \eta\right)\right)$. By Lemma 2.8 and (2.8), $v_{\varepsilon}^{(i)} \in$ $\partial f(p)$. Hence, $\tau_{p}^{q} \nabla f_{\varepsilon}{ }^{\left(p_{i}\right)}(q) \in \partial f(p)_{\eta}$ for all $q \in \operatorname{supp} \psi_{i} \cap B_{\delta_{1}(p, \eta)}(p)$, and $\varepsilon \in\left(0, \varepsilon\left(p, p_{i}, \eta\right)\right)$.

Lemma 2.11 For any $\eta>0$, there exist numbers $\delta_{2}(p, \eta)>0$ and $\varepsilon(p, \eta)>0$ such that

$$
\tau_{p}^{q} \nabla f_{\varepsilon}(q) \in \partial f(p)_{\eta}
$$

for all $q \in B_{\delta_{2}(p, \eta)}(p)$ and all $\varepsilon \in(0, \varepsilon(p, \eta))$.

Proof. By Lemma 2.10, $\tau_{p}^{q} \nabla f_{\varepsilon}^{\left(p_{i}\right)}(q) \in \partial f(p)_{\eta / 2}$ holds for all $q \in B_{\delta_{1}(p, \eta / 2)}(p) \cap \operatorname{supp} \psi_{i}$ and $\varepsilon \in\left(0, \varepsilon\left(p, p_{i}, \eta / 2\right)\right)$. Since $\partial f(p)_{\eta / 2}$ is convex,

$$
\sum_{i} \psi_{i}(q) \cdot \tau_{p}^{q} \nabla f_{\varepsilon}^{\left(p_{i}\right)}(q) \in \partial f(p)_{\eta / 2}
$$

for all $q \in B_{\delta_{1}(p, \eta / 2)}(p)$ and $\varepsilon \in\left(0, \varepsilon_{1}(p, \eta)\right)$, where

$$
\varepsilon_{1}(p, \eta):=\min \left\{\varepsilon\left(p, p_{i}, \eta / 2\right) \mid B_{\delta_{1}(p, \eta / 2)}(p) \cap \operatorname{supp} \psi_{i} \neq \emptyset\right\} .
$$

Fix any $q \in B_{\delta_{2}(p, \eta)}(p)$, where $\delta_{2}(p, \eta):=\delta_{1}(p, \eta / 2)$, and take any $\tilde{u} \in \mathbb{S}_{q}^{n-1}$. Since $\sum_{i} \psi_{i}=1, \sum_{i} d \psi_{i}(\tilde{u})=0$ holds. Then, we get

$$
\left(d f_{\varepsilon}\right)_{q}(\tilde{u})=\sum_{i} \psi_{i}(q) d f_{\varepsilon}^{\left(p_{i}\right)}(\tilde{u})+\sum_{i} d \psi_{i}(\tilde{u})\left(f_{\varepsilon}^{\left(p_{i}\right)}(q)-f(q)\right)
$$

By Lemma 2.2.

$$
\left|\sum_{i} d \psi_{i}(\tilde{u})\left(f_{\varepsilon}^{\left(p_{i}\right)}(q)-f(q)\right)\right| \leq \varepsilon \sum_{i}\left|d \psi_{i}(\tilde{u})\right| \cdot \operatorname{Lip}(f) \cdot \operatorname{Lip}\left(\exp _{p_{i}} \mid \mathbb{B}_{r_{i}}\left(o_{p_{i}}\right)\right)
$$

holds. Therefore, there exists a number $\varepsilon(p, \eta) \in\left(0, \varepsilon_{1}(p, \eta)\right]$ such that

$$
\left|\sum_{i} d \psi_{i}(\tilde{u})\left(f_{\varepsilon}^{\left(p_{i}\right)}(q)-f(q)\right)\right|<\frac{\eta}{2}
$$

for all $\varepsilon \in(0, \varepsilon(p, \eta))$ and for all $q \in B_{\delta_{2}(p, \eta)}(p)$. By (2.13) and (2.14),

$$
\left|\left\langle\nabla f_{\varepsilon}(q), \tilde{u}\right\rangle-\left\langle\sum_{i} \psi_{i}(q) \nabla f_{\varepsilon}^{\left(p_{i}\right)}(q), \tilde{u}\right\rangle\right|<\frac{\eta}{2}
$$


If we set $u:=\tau_{p}^{q} \tilde{u}$, then

$$
\left|\left\langle\tau_{p}^{q} \nabla f_{\varepsilon}(q), u\right\rangle-\left\langle\sum_{i} \psi_{i}(q) \cdot \tau_{p}^{q} \nabla f_{\varepsilon}^{\left(p_{i}\right)}(q), u\right\rangle\right|<\frac{\eta}{2} .
$$

Since $u$ is any unit tangent vector,

$$
\left\|\tau_{p}^{q} \nabla f_{\varepsilon}(q)-\sum_{i} \psi_{i}(q) \cdot \tau_{p}^{q} \nabla f_{\varepsilon}^{\left(p_{i}\right)}(q)\right\|<\frac{\eta}{2} .
$$

Hence, from (2.12), it follows that $\tau_{p}^{q} \nabla f_{\varepsilon}(q) \in \partial f(p)_{\eta}$ for all $q \in B_{\delta_{2}(p, \eta)}(p)$ and all $\varepsilon \in(0, \varepsilon(p, \eta))$.

Lemma 2.12 Let $A, B \subset \mathbb{R}^{n} \backslash\{o\}$ be compact sets such that $\angle(u, v)>\pi / 2$ holds for all $u \in A$ and $v \in B$. Then, $o \notin \operatorname{Conv}(A)$ and $o \notin \operatorname{Conv}(B)$. Moreover, $\angle(u, v)>\pi / 2$ also holds for all $u \in \operatorname{Conv}(A)$ and $v \in \operatorname{Conv}(B)$.

Proof. Take any $u \in A$, and fix it. Since $\angle(u, v)>\pi / 2$ for all $v \in B, B$ is a subset of the open convex cone $V_{u}:=\left\{v \in \mathbb{R}^{n} \backslash\{o\} \mid \angle(u, v)>\pi / 2\right\}$. Thus, $B \subset \bigcap_{u \in A} V_{u}$. The set $\bigcap_{u \in A} V_{u}$ is convex, since each $V_{u}$ is convex. From the definition of the convex hull, $\operatorname{Conv}(B) \subset \bigcap_{u \in A} V_{u}$. Hence, we have proved that $o \notin \operatorname{Conv}(B)$, and that $\angle(u, v)>\pi / 2$ for all $u \in A$ and all $v \in \operatorname{Conv}(B)$. On the other hand, choose any $v \in \operatorname{Conv}(B)$, and fix it. Since $\angle(u, v)>\pi / 2$ for all $u \in A, A$ is a subset of the open convex cone $V_{v}$. Then, $A \subset \bigcap_{v \in \operatorname{Conv}(B)} V_{v}$, and hence $\operatorname{Conv}(A) \subset \bigcap_{v \in \operatorname{Conv}(B)} V_{v}$. In particular, $o \notin \operatorname{Conv}(A)$ and $\angle(u, v)>\pi / 2$ for all $u \in \operatorname{Conv}(A)$ and all $v \in \operatorname{Conv}(B)$.

Lemma 2.13 Let $f$ and $h$ be Lipschitz functions on $M$. Assume that $o \notin \partial f(x)$, o $\notin$ $\partial h(x)$ on a compact set $K \subset M$. If $\angle(u, v)>\pi / 2$ holds for all $x \in K$ and all $u \in \partial f(x)$, $v \in \partial h(x)$, then there exists a number $\eta_{K}>0$ such that, for any $x \in K$ and any $u \in$ $\partial f(x)_{\eta_{K}}, v \in \partial h(x)_{\eta_{K}}, \angle(u, v)>\pi / 2$ holds.

Proof. By supposing that the conclusion is false, we will get a contradiction. Then, for each positive integer $i$, there exist a point $x_{i} \in K, u_{i} \in \partial f\left(x_{i}\right)$, and $v_{i} \in \partial h\left(x_{i}\right)$ such that $\angle\left(u_{i}, v_{i}\right)<\pi / 2+1 / i$. Since $K$ is compact, we can assume, taking a subsequence, if necessary, that $x:=\lim _{i \rightarrow \infty} x_{i} \in K$ exists. By definition, any limits of the sequences $\left\{\tau_{x}^{x_{i}} u_{i}\right\}$ and $\left\{\tau_{x}^{x_{i}} v_{i}\right\}$ are elements of $\partial f(x)$ and $\partial h(x)$, respectively. Since $\angle\left(\tau_{x}^{x_{i}} u_{i}, \tau_{x}^{x_{i}} v_{i}\right)=$ $\angle\left(u_{i}, v_{i}\right)<\pi / 2+1 / i$ for each $i$, we get $\angle(u, v) \leq \pi / 2$ for some $u \in \partial f(x)$ and $v \in \partial h(x)$. This is a contradiction.

Lemma 2.14 Let $K \subset M$ be a compact set. Assume that there exist two distinct points $p, q \in M \backslash K$ such that for any minimal geodesic segments $\alpha$ and $\beta$ emanating from each point $x \in K$ to $p$ and $q$, respectively, $\angle(\dot{\alpha}(0), \dot{\beta}(0))>\pi / 2$ holds. Then, for any sufficiently small $\varepsilon>0, \angle\left(\nabla\left(d_{p}\right)_{\varepsilon}, \nabla\left(d_{q}\right)_{\varepsilon}\right)>\pi / 2$ holds on $K$, where $d_{p}(x):=d(p, x)$ for all $x \in M$.

Proof. Let $x$ be any point of $K$. It follows from Lemma 2.12 that $o \notin \partial d_{p}(x) \cup \partial d_{q}(x)$ and $\angle(u, v)>\pi / 2$ holds for all $u \in \partial d_{p}(x)$ and $v \in \partial d_{q}(x)$. Then, by Lemma 2.13, 
there exists a positive number $\eta_{K}$ such that, for any $x \in K, u \in \partial d_{p}(x)_{\eta_{K}}$, and $v \in$ $\partial d_{q}(x)_{\eta_{K}}, \angle(u, v)>\pi / 2$ holds again. It follows from Lemma 2.11 that, for each point $x \in K$, there exist two numbers $\delta_{2}\left(x, \eta_{K}\right)>0$ and $\varepsilon\left(x, \eta_{K}\right)>0$ such that $\tau_{x}^{q_{1}} \nabla\left(d_{p}\right)_{\varepsilon}\left(q_{1}\right) \in$ $\partial d_{p}(x)_{\eta_{K}}$ and $\tau_{x}^{q_{1}} \nabla\left(d_{q}\right)_{\varepsilon}\left(q_{1}\right) \in \partial d_{q}(x)_{\eta_{K}}$ for all $q_{1} \in B_{\delta_{2}\left(x, \eta_{K}\right)}(x)$ and $\varepsilon \in\left(0, \varepsilon\left(x, \eta_{K}\right)\right)$. Since $\angle\left(\nabla\left(d_{p}\right)_{\varepsilon}\left(q_{1}\right), \nabla\left(d_{q}\right)_{\varepsilon}\left(q_{1}\right)\right)=\angle\left(\tau_{x}^{q_{1}} \nabla\left(d_{p}\right)_{\varepsilon}\left(q_{1}\right), \tau_{x}^{q_{1}} \nabla\left(d_{q}\right)_{\varepsilon}\left(q_{1}\right)\right)$, it follows from Lemma 2.13 that $\angle\left(\nabla\left(d_{p}\right)_{\varepsilon}\left(q_{1}\right), \nabla\left(d_{q}\right)_{\varepsilon}\left(q_{1}\right)\right)>\pi / 2$ for all $q_{1} \in B_{\delta_{2}\left(x, \eta_{K}\right)}(x)$ and all $\varepsilon \in\left(0, \varepsilon\left(x, \eta_{K}\right)\right)$. This implies that, for any sufficiently small $\varepsilon>0, \angle\left(\nabla\left(d_{p}\right)_{\varepsilon}, \nabla\left(d_{q}\right)_{\varepsilon}\right)>\pi / 2$ holds on $K$ since $K$ is compact.

\subsection{Approximations of Lipschitz maps: Proof of Theorem 1.3}

In this subsection, using some techniques from non-smooth analysis and the partition of unity, we first treat an approximation of a Lipschitz map $F$ from a compact Riemannian manifold $M$ of dimension $n$ into a Riemannian manifold $N$ of dimension $k$, and finally prove Theorem 1.3 applying the approximation method.

Let $N$ be embedded into Euclidean $m$-dimensional space $\left(\mathbb{R}^{m},\langle\cdot, \cdot\rangle\right)$, where $m \geq k+1$. We may assume that $N$ is isometrically embedded into $\mathbb{R}^{m}$ by introducing the induced metric from the space. Here, note that the notion of the singular point of a Lipschitz map is independent of the choice of the Riemannian metric (See (1.1) and Definition 1.5). The Lipschitz map $F$ is therefore a map from $M$ into $\mathbb{R}^{m}$. Then, we may define a smooth approximation of $F$ on a convex ball $B_{r}(p)$ of radius $r$, centered at each point $p \in M$.

Definition 2.15 For each $\varepsilon>0$, let $F_{\varepsilon}^{(p)}: B_{r}(p) \longrightarrow \mathbb{R}^{m}$ denote the map defined by

$$
F_{\varepsilon}^{(p)}(q):=\int_{\mathbb{R}^{n}} \rho_{\varepsilon}(y) F(q(y)) d y=\int_{\mathbb{R}^{n}} \rho_{\varepsilon}\left(\exp _{p}^{-1} q-y\right) F\left(\exp _{p}(y)\right) d y,
$$

where $q(y):=\exp _{p}\left(\exp _{p}^{-1} q-y\right)$ and $\rho_{\varepsilon}$ denotes the mollifier.

Lemma 2.16 For any $\varepsilon>0$ and any $q \in B_{r}(p)$,

$$
\left\|F_{\varepsilon}^{(p)}(q)-F(q)\right\| \leq \varepsilon \cdot \operatorname{Lip}(F) \cdot \operatorname{Lip}\left(\left.\exp _{p}\right|_{\mathbb{B}_{r+\varepsilon}\left(o_{p}\right)}\right)
$$

holds, where $\|\cdot\|$ denotes the Euclidean norm of $\mathbb{R}^{m}$.

Proof. After the fashion of the proof of Lemma 2.2, we have the desired inequality.

Since $M$ is compact, we can choose finitely many convex balls $B_{r_{i}}\left(p_{i}\right), i=1,2, \ldots, \ell$, which cover $M$. Take a partition of unity $\varphi_{i}$ subordinate to $\left\{B_{r_{i}}\left(p_{i}\right)\right\}$. Then, for each $\varepsilon>0$, we define the global approximation $F_{\varepsilon}$ of $F$ by

$$
F_{\varepsilon}(q)=\sum_{i=1}^{\ell} \varphi_{i}(q) F_{\varepsilon}^{\left(p_{i}\right)}(q)
$$

Lemma 2.17 For any $\varepsilon>0$ and any $q \in M$, we have

$$
\left\|F_{\varepsilon}(q)-F(q)\right\| \leq \varepsilon \cdot \operatorname{Lip}(F) \sum_{i=1}^{\ell} \varphi_{i}(q) \operatorname{Lip}\left(\left.\exp _{p_{i}}\right|_{\mathbb{B}_{r_{i}+\varepsilon}\left(o_{p_{i}}\right)}\right)
$$


Proof. Since $\sum_{i=1}^{\ell} \varphi_{i}=1$, we get $F(q)=\sum_{i=1}^{\ell} \varphi_{i}(q) F(q)$. Hence, by Lemma 2.16 and the triangle inequality, we have the desired inequality.

In what follows, for a pair of points $p$ and $q$ of $M$ or $N$ admitting a unique minimal geodesic segment $\gamma$, we denote by $\tau_{q}^{p}$ the parallel transport from the tangent space at $p$ onto the tangent space at $q$ along $\gamma$.

Lemma 2.18 Let $q \in \operatorname{supp} \varphi_{i}$. Then, for any $\tilde{u} \in \mathbb{S}_{q}^{n-1}:=\left\{v \in T_{q} M \mid\|v\|=1\right\}$,

$$
\left\|\left(d F_{\varepsilon}^{\left(p_{i}\right)}\right)_{q}(\tilde{u})\right\| \leq \operatorname{Lip}(F)\left(1+\sup _{y \in \mathbb{B}_{\varepsilon}\left(o_{p_{i}}\right)}\left\|Y_{y}^{(\tilde{u})}(1)-\tau_{q_{i}(y)}^{q}(\tilde{u})\right\|\right)
$$

holds, where $q_{i}(y):=\exp _{p_{i}}\left(\exp _{p_{i}}^{-1} q-y\right)$, and $Y_{y}^{(\tilde{u})}(t):=\left.\frac{\partial}{\partial s} \exp _{p_{i}} t\left(\exp _{p_{i}}^{-1}\left(\exp _{q} s \tilde{u}\right)-y\right)\right|_{s=0}$ is a Jacobi field along the geodesic $\exp _{p_{i}} t\left(\exp _{p_{i}}^{-1} q-y\right)$ for each $y$.

Proof. From (2.15), it is easy to obtain

$$
\left(d F_{\varepsilon}^{\left(p_{i}\right)}\right)_{q}(\tilde{u})=\int_{\mathbb{R}^{n}} \rho_{\varepsilon}(y) d F_{q_{i}(y)}\left(Y_{y}^{(\tilde{u})}(1)\right) d y
$$

and $d F_{q_{i}(y)}\left(Y_{y}^{(\tilde{u})}(1)\right)=d F_{q_{i}(y)}\left(Y_{y}^{(\tilde{u})}(1)-\tau_{q_{i}(y)}^{q}(\tilde{u})\right)+d F_{q_{i}(y)}\left(\tau_{q_{i}(y)}^{q}(\tilde{u})\right)$. Hence, by the triangle inequality, we get (2.17).

Lemma 2.19 For any $\eta>0$, there exists a number $\varepsilon_{i}(\eta)>0$ such that

$$
\sup \left\{\left\|Y_{y}^{(\tilde{u})}(1)-\tau_{q_{i}(y)}^{q}(\tilde{u})\right\| \mid q \in \operatorname{supp} \varphi_{i}, \tilde{u} \in \mathbb{S}_{q}^{n-1}, y \in \mathbb{B}_{\varepsilon_{i}(\eta)}\left(o_{p_{i}}\right)\right\}<\eta .
$$

Proof. In a similar way to the proof of Lemma 2.5, we get the inequality.

Lemma 2.20 For any $\eta>0$, there exists a number $\varepsilon(\eta)>0$ such that

$$
\left\|d F_{\varepsilon}(\tilde{u})\right\| \leq(1+\eta) \operatorname{Lip}(F)
$$

holds for all $\varepsilon \in(0, \varepsilon(\eta))$ and all unit tangent vectors $\tilde{u}$ on $M$.

Proof. Take any $\tilde{u} \in \mathbb{S}_{q}^{n-1}$, and fix it. Since $\sum_{i=1}^{\ell} \varphi_{i}=1$ on $M$, we get $\sum_{i=1}^{\ell}\left(d \varphi_{i}\right)_{q}(\tilde{u})=0$. Then, we have

$$
\left(d F_{\varepsilon}\right)_{q}(\tilde{u})=\sum_{i=1}^{\ell} \varphi_{i}(q)\left(d F_{\varepsilon}^{\left(p_{i}\right)}\right)_{q}(\tilde{u})+\sum_{i=1}^{\ell}\left(d \varphi_{i}\right)_{q}(\tilde{u})\left(F_{\varepsilon}^{\left(p_{i}\right)}(q)-F(q)\right) .
$$

By applying the triangle inequality to the equation above, we have

$$
\left\|\left(d F_{\varepsilon}\right)_{q}(\tilde{u})\right\| \leq \sum_{i=1}^{\ell} \varphi_{i}(q)\left\|\left(d F_{\varepsilon}^{\left(p_{i}\right)}\right)_{q}(\tilde{u})\right\|+\sum_{i=1}^{\ell}\left|\left(d \varphi_{i}\right)_{q}(\tilde{u})\right| \cdot\left\|F_{\varepsilon}^{\left(p_{i}\right)}(q)-F(q)\right\| .
$$

By Lemmas 2.16, 2.18 and 2.19, we get (2.19) for all sufficiently small $\varepsilon>0$.

From now on, we assume that $n=\operatorname{dim} M \leq \operatorname{dim} N=k$. 
Lemma 2.21 For each non-singular point $p \in M$ of $F$, there exist positive numbers $r(p)$ and $\delta(p)$ such that, for any $u \in \mathbb{S}_{p}^{n-1}$, there exists a local unit vector field $V$ on a neighborhood of $F(p)$ satisfying

$$
\left\langle d F_{q}\left(\tau_{q}^{p}(u)\right), V_{F(q)}\right\rangle \geq \delta(p)
$$

for almost all $q \in B_{2 r(p)}(p)$.

Proof. Choose convex balls $B_{r_{1}}(p)$ and $B_{r_{2}}(F(p))$ so as to satisfy $F\left(B_{r_{1}}(p)\right) \subset B_{r_{2}}(F(p))$. Since the point $p$ is a non-singular point of $F$, it follows from Example 1.8 that there exist positive numbers $r(p) \in\left(0, r_{1} / 2\right)$ and $\delta(p)$ satisfying the following property: For any $u \in \mathbb{S}_{p}^{n-1}$ at the point $p$, there exists a unit vector $v$ at $F(p)$ such that

$$
\left\langle\tau_{F(p)}^{F(q)} \circ d F_{q} \circ \tau_{q}^{p}(u), v\right\rangle \geq \delta(p)
$$

for almost all $q \in B_{2 r(p)}(p)$. Hence, we get $\left\langle d F_{q}\left(\tau_{q}^{p}(u)\right), V_{F(q)}\right\rangle \geq \delta(p)$ for almost all $q \in B_{2 r(p)}(p)$, where $V_{F(q)}:=\tau_{F(q)}^{F(p)}(v)$.

Henceforth, we fix any non-singular point $p \in M$ of $F$ and any $\tilde{u} \in \mathbb{S}_{q}^{n-1}$ at any point $q \in B_{r(p)}(p)$. Here, we also fix an integer $i \in\{1,2, \ldots, \ell\}$ satisfying $q \in \operatorname{supp} \varphi_{i} \subset B_{r_{i}}\left(p_{i}\right)$.

Lemma 2.22 There exists a unit vector field $V$ on a neighborhood of $F(p)$ such that

$$
\begin{aligned}
& \left\langle\left(d F_{\varepsilon}^{\left(p_{i}\right)}\right)_{q}(\tilde{u}), V_{F(q)}\right\rangle \\
& \geq-\operatorname{Lip}(F)\left(\sup _{y \in \mathbb{B}_{\varepsilon}\left(o_{p_{i}}\right)}\left\|Y_{y}^{(\tilde{u})}(1)-U_{q_{i}(y)}\right\|+\sup _{y \in \mathbb{B}_{\varepsilon}\left(o_{p_{i}}\right)}\left\|V_{F(q)}-V_{F\left(q_{i}(y)\right)}\right\|\right)+\delta(p)
\end{aligned}
$$

for all $\varepsilon \in\left(0, \varepsilon_{i}(p)\right)$. Here, $\varepsilon_{i}(p):=\min \left\{r_{i}, r(p) / \operatorname{Lip}\left(\exp _{p_{i}} \mid \mathbb{B}_{2 r_{i}}\left(o_{p_{i}}\right)\right)\right\}$ and $U_{q_{i}(y)}:=\tau_{q_{i}(y)}^{p} \circ$ $\tau_{p}^{q}(\tilde{u})$

Proof. It follows from Lemma 2.21 that for the unit tangent vector $u:=\tau_{p}^{q}(\tilde{u})$, there exists a unit vector field $V$ on a neighborhood of $F(p)$ satisfying (2.21). By the triangle inequality, $\left\|\exp _{p_{i}}^{-1} q-y\right\|<r_{i}+\|y\|<2 r_{i}$ for all $y \in \mathbb{B}_{r_{i}}\left(o_{p_{i}}\right)$. Thus, we get

$$
d_{M}\left(q, q_{i}(y)\right) \leq\|y\| \cdot \operatorname{Lip}\left(\left.\exp _{p_{i}}\right|_{\mathbb{B}_{2 r_{i}}\left(o_{p_{i}}\right)}\right)
$$

for all $y \in \mathbb{B}_{r_{i}}\left(o_{p_{i}}\right)$, where $d_{M}$ denotes the distance function of $M$. Then, from the triangle inequality, we obtain

$$
d_{M}\left(p, q_{i}(y)\right) \leq d_{M}(p, q)+d_{M}\left(q, q_{i}(y)\right)<2 r(p)
$$

for all $y \in \mathbb{B}_{\varepsilon_{i}(p)}\left(o_{p_{i}}\right)$. Thus, by Lemma 2.21,

$$
\left\langle d F_{q_{i}(y)}\left(U_{q_{i}(y)}\right), V_{F\left(q_{i}(y)\right)}\right\rangle \geq \delta(p)
$$

for almost all $y \in \mathbb{B}_{\varepsilon_{i}(p)}\left(o_{p_{i}}\right)$, where $U_{q_{i}(y)}:=\tau_{q_{i}(y)}^{p}(u)$. It is clear to see that for almost all $y \in \mathbb{B}_{\varepsilon}\left(o_{p_{i}}\right)$,

$$
\begin{aligned}
& \left\langle d F_{q_{i}(y)}\left(U_{q_{i}(y)}\right), V_{F(q)}\right\rangle \\
& =\left\langle d F_{q_{i}(y)}\left(U_{q_{i}(y)}\right), V_{F(q)}-V_{F\left(q_{i}(y)\right)}\right\rangle+\left\langle d F_{q_{i}(y)}\left(U_{q_{i}(y)}\right), V_{F\left(q_{i}(y)\right)}\right\rangle \\
& \geq-\operatorname{Lip}(F) \sup _{y \in \mathbb{B}_{\varepsilon}\left(o_{p_{i}}\right)}\left\|V_{F(q)}-V_{F\left(q_{i}(y)\right)}\right\|+\left\langle d F_{q_{i}(y)}\left(U_{q_{i}(y)}\right), V_{F\left(q_{i}(y)\right)}\right\rangle .
\end{aligned}
$$


Since $d F_{q_{i}(y)}\left(Y_{y}^{(\tilde{u})}(1)\right)=d F_{q_{i}(y)}\left(Y_{y}^{(\tilde{u})}(1)-U_{q_{i}(y)}\right)+d F_{q_{i}(y)}\left(U_{q_{i}(y)}\right)$, we therefore get (2.22) from (2.18), (2.23) and (2.24).

Lemma 2.23 For any $\eta>0$, there exists a number $\varepsilon_{i}(p, \eta)>0$ such that

$$
\sup \left\{\left\|Y_{y}^{(\tilde{u})}(1)-U_{q_{i}(y)}\right\| \mid q \in \operatorname{supp} \varphi_{i} \cap \overline{B_{r(p)}(p)}, \tilde{u} \in \mathbb{S}_{q}^{n-1}, y \in \mathbb{B}_{\varepsilon_{i}(p, \eta)}\left(o_{p_{i}}\right)\right\}<\eta
$$

and

$$
\sup \left\{\left\|\tau_{F(q)}^{F(p)}(v)-\tau_{F\left(q_{i}(y)\right)}^{F(p)}(v)\right\| \mid q \in \operatorname{supp} \varphi_{i} \cap \overline{B_{r(p)}(p)}, v \in \mathbb{S}_{F(p)}^{k-1}, y \in \mathbb{B}_{\varepsilon_{i}(p, \eta)}\left(o_{p_{i}}\right)\right\}<\eta,
$$

where $\overline{B_{r(p)}(p)}:=\{q \in M \mid d(p, q) \leq r(p)\}$.

Proof. Imitate the proof of Lemma 2.19.

Lemma 2.24 There exists $V_{F(q)} \in \mathbb{S}_{F(q)}^{k-1}$ such that

$$
\left\langle\left(d F_{\varepsilon}^{\left(p_{i}\right)}\right)_{q}(\tilde{u}), V_{F(q)}\right\rangle \geq \frac{2 \delta(p)}{3}
$$

for all $\varepsilon \in(0, \varepsilon(p))$ and all $p_{i}$ with $q \in \operatorname{supp} \varphi_{i}$. Here we set

$$
\varepsilon(p):=\min \left\{\varepsilon_{i}(p), \varepsilon_{i}\left(p, \eta_{0}\right) \mid \operatorname{supp} \varphi_{i} \cap B_{r(p)}(p) \neq \emptyset\right\},
$$

where $\eta_{0}:=\delta(p) / 6 \operatorname{Lip}(F)$.

Proof. The inequality is immediate from Lemmas 2.22 and 2.23 ,

Lemma 2.25 There exist $V_{F(q)} \in \mathbb{S}_{F(q)}^{k-1}$ and a number $\varepsilon_{0}(p)>0$ such that

$$
\left\langle\left(d F_{\varepsilon}\right)_{q}(\tilde{u}), V_{F(q)}\right\rangle \geq \frac{1}{3} \delta(p)
$$

for all $\varepsilon \in\left(0, \varepsilon_{0}(p)\right)$.

Proof. By Lemma 2.24, for any $\varepsilon \in(0, \varepsilon(p))$,

$$
\sum_{i=1}^{\ell} \varphi_{i}(q)\left\langle d F_{\varepsilon}^{\left(p_{i}\right)}(\tilde{u}), V_{F(q)}\right\rangle \geq \frac{2}{3} \delta(p) .
$$

From Lemma 2.16, we may choose a number $\varepsilon_{0}(p) \in(0, \varepsilon(p))$ satisfying

$$
\left|\sum_{i=1}^{\ell} d \varphi_{i}(\tilde{u})\left\langle F_{\varepsilon}^{\left(p_{i}\right)}(q)-F(q), V_{F(q)}\right\rangle\right|<\frac{1}{3} \delta(p)
$$

for all $\varepsilon \in\left(0, \varepsilon_{0}(p)\right)$. Combining (2.20), (2.26) and (2.27), we get (2.25). 
(Proof of Theorem 1.3) Let $F: M \longrightarrow N$ be a Lipschitz map from a compact Riemannian manifold $M$ into a Riemannian manifold $N$, where $\operatorname{dim} M \leq \operatorname{dim} N$. Assume that $F$ has no singular points on $M$. We embed $N$ into $\mathbb{R}^{m}$, where $m \geq \operatorname{dim} N+1$, and introduce the induced metric from the outer space to $N$. Note that $F: M \longrightarrow \mathbb{R}^{m}$. Since $N$ is a smooth submanifold in $\mathbb{R}^{m}$, for any $x \in N$ there exists an open neighborhood $U_{x}$ of $x$ in $\mathbb{R}^{m}$ such that each $y \in U_{x}$ admits a unique $z \in N$ with $\|y-z\|=\inf _{w \in N}\|y-w\|$. Considering the open set $\mathcal{U}_{N}:=\cup_{x \in N} U_{x}$ in $\mathbb{R}^{m}$, we have the smooth locally distance projection $\pi_{N}: \mathcal{U}_{N} \longrightarrow N$. Note here that $N \subset \mathcal{U}_{N}$. Since $F(M)$ is compact, it follows from Lemma 2.17 that for all sufficiently small $\varepsilon>0$, the image of the map $F_{\varepsilon}$ defined by (2.16) is a subset of $\mathcal{U}_{N}$. Then, for any sufficiently small $\varepsilon>0$, we can define the smooth map $\pi_{N} \circ F_{\varepsilon}$ from $M$ into $N$. By its definition, $\left(d \pi_{N}\right)_{x}$ is an orthogonal projection to $T_{x} N$ for each $x \in N$. Therefore, for any sufficiently small $\varepsilon>0$, the map $\pi_{N} \circ F_{\varepsilon}: M \longrightarrow N$ is an immersion from Lemma 2.25. Combining Lemmas 2.17, 2.20 and the argument above, we get the assertion.

\subsection{Proof of Corollary 1.10}

We need one more lemma in order to prove Corollary 1.10, In what follows, $d_{M}$ denotes the distance function of a given Riemannian manifold $M$.

Lemma 2.26 Let $M$ be a compact (connected) Riemannian manifold, and let $F: M \longrightarrow$ $M$ be a local diffeomorphism. If $\max _{x \in M} d_{M}(F(x), x)$ is sufficiently small, then $F$ is injective. In particular, $F$ is a diffeomorphism.

Proof. Since $F(M)$ is open and closed in $M, F$ is surjective. Then, we can consider $F$ to be a covering map. Since $M$ is compact, there exists a number $a$ such that $0<a<i(M)$, where $i(M)$ denotes the injectivity radius of $M$. Since $\max _{x \in M} d_{M}(F(x), x) \ll 1$, we may assume that $d_{M}(F(x), x)<i(M)$ for all $x \in M$. Thus, for each $x \in M$, there exists a unique minimal geodesic segment $\gamma:[0,1] \longrightarrow M$ joining $x=\gamma(0)$ to $F(x)=\gamma(1)$. Define the map $H: M \times[0,1] \longrightarrow M$ by $H(x, t):=\sigma_{x}(t)$, where $\sigma_{x}:[0,1] \longrightarrow M$ denotes a unique minimal geodesic segment emanating from $x=\sigma_{x}(0)$ to $F(x)=\sigma_{x}(1)$. $H$ is continuous, for $F$ is continuous and $\sigma_{x}$ is unique. By the definition of $H, H(x, 0)=$ $x=\operatorname{id}_{M}(x)$ and $H(x, 1)=F(x)$ for all $x \in M$, i.e., $F$ is homotopic to $\mathrm{id}_{M}$. Since $\pi_{1}(M, F(x)) / F_{\sharp}\left(\pi_{1}(M, x)\right)$ is trivial, where $F_{\sharp}$ denotes the homomorphism from $\pi_{1}(M, x)$ to $\pi_{1}(M, F(x))$ induced by $F$, it follows from a well known lemma (cf. Corollary 3 in [33, Chapter 3]) that $F^{-1}(x)$ is one point, i.e., $F$ is injective.

(Proof of Corollary 1.10) Let $F$ be a bi-Lipschitz homeomorphism from a compact Riemannian manifold $M$ onto a Riemannian manifold $N$. Assume that $F$ and $F^{-1}$ have no singular points on $M$ and $N$, respectively. By Theorem 1.3, for any $\eta>0$, there exist two smooth immersions $f_{\eta}$ from $M$ into $N$ and $g_{\eta}$ from $N$ into $M$ such that

$$
\max _{p \in M} d_{N}\left(f_{\eta}(p), F(p)\right)<\eta, \quad \operatorname{Lip}\left(f_{\eta}\right) \leq \operatorname{Lip}(F)(1+\eta)
$$

and that

$$
\max _{q \in N} d_{M}\left(g_{\eta}(q), F^{-1}(q)\right)<\eta, \quad \operatorname{Lip}\left(g_{\eta}\right) \leq \operatorname{Lip}\left(F^{-1}\right)(1+\eta)
$$


respectively. Hence, $\operatorname{Lip}\left(g_{\eta} \circ f_{\eta}\right) \leq \operatorname{Lip}\left(g_{\eta}\right) \operatorname{Lip}\left(f_{\eta}\right) \leq \operatorname{Lip}(F) \operatorname{Lip}\left(F^{-1}\right)(1+\eta)^{2}$. Moreover, by the triangle inequality, we get

$$
d_{M}\left(g_{\eta} \circ f_{\eta}(p), p\right) \leq d_{M}\left(g_{\eta}\left(f_{\eta}(p)\right), F^{-1}\left(f_{\eta}(p)\right)\right)+d_{M}\left(F^{-1}\left(f_{\eta}(p)\right), F^{-1}(F(p))\right) .
$$

Since $d_{M}\left(g_{\eta}\left(f_{\eta}(p)\right), F^{-1}\left(f_{\eta}(p)\right)\right)<\eta$ and $d_{M}\left(F^{-1}\left(f_{\eta}(p)\right), F^{-1}(F(p))\right)<\eta \operatorname{Lip}\left(F^{-1}\right)$ for all $p \in M$, we obtain $\max _{p \in M} d_{M}\left(g_{\eta} \circ f_{\eta}(p), p\right)<\eta\left(1+\operatorname{Lip}\left(F^{-1}\right)\right)$. Therefore, by Lemma 2.26, for all sufficiently small $\eta>0, g_{\eta} \circ f_{\eta}$ is a diffeomorphism on $M$. This implies that $f_{\eta}$ and $g_{\eta}$ are injective for all sufficiently small $\eta$, and hence $M$ and $N$ are diffeomorphic.

\section{Proofs of the differentiable exotic sphere theorems}

We first give two preliminaries to three cases (1.6), (1.7), and (1.10).

\subsection{Preliminaries to the proofs in assuming (1.6) and (1.10)}

Throughout this subsection, let $\sigma: S^{n-1}(1):=\left\{v \in \mathbb{R}^{n} \mid\|v\|=1\right\} \longrightarrow S^{n-1}(1)$ be a diffeomorphism satisfying

$$
\int_{0}^{\pi} e^{-t}\|\ddot{c}(t)+c(t)\| d t \leq e^{-\pi} \alpha
$$

for some $\alpha>0$ and any unit speed geodesic $\gamma:[0, \pi] \longrightarrow S^{n-1}(1)$. Here,

$$
c:=\sigma \circ \gamma, \quad \dot{c}:=\frac{d c}{d t}, \quad \text { and } \quad \ddot{c}:=\frac{d^{2} c}{d t^{2}} .
$$

Our aim in this subsection is to prove the following theorem:

Theorem 3.1 If $\alpha>0$ is sufficiently small, for example $\alpha=1-1 / \sqrt{2}$, then the origin $o \in \mathbb{R}^{n}$ is non-singular for the map $\widetilde{F}: \mathbb{R}^{n} \longrightarrow \mathbb{R}^{n}$ defined by

$$
\widetilde{F}(v):= \begin{cases}\|v\| \sigma\left(\frac{v}{\|v\|}\right) & \text { on } \mathbb{R}^{n} \backslash\{o\} \\ o & \text { when } v=o .\end{cases}
$$

We need six lemmas to prove Theorem 3.1. So, we first prove the lemmas before proving the theorem: Take any unit speed geodesic $\gamma:[0, \pi] \longrightarrow S^{n-1}(1)$, and fix it. Set

$$
\bar{c}(t):=c(0) \cos t+\dot{c}(0) \sin t
$$

on $[0, \pi]$.

Lemma 3.2 For any $t \in[0, \pi]$,

$$
\sqrt{\|c(t)-\bar{c}(t)\|^{2}+\|\dot{c}(t)-\dot{\bar{c}}(t)\|^{2}} \leq e^{\pi} \int_{0}^{\pi} e^{-\theta}\|\ddot{c}(\theta)+c(\theta)\| d \theta
$$


holds. In particular, we have

$$
\|c(t)-\bar{c}(t)\| \leq \alpha
$$

for all $t \in[0, \pi]$.

Proof. Let $f(t):=\sqrt{\|c(t)-\bar{c}(t)\|^{2}+\|\dot{c}(t)-\dot{\bar{c}}(t)\|^{2}}$, and let $X(t):=\left(\begin{array}{c}c(t) \\ \dot{c}(t)\end{array}\right), \bar{X}(t):=$ $\left(\begin{array}{c}\bar{c}(t) \\ \dot{\bar{c}}(t)\end{array}\right) \in \mathbb{R}^{2 n}$. Then, $f(t)=\|X(t)-\bar{X}(t)\|$. Note that $f(0)=0$ because $\bar{c}(0)=c(0)$ and $\dot{\bar{c}}(0)=\dot{c}(0)$. Choose an open interval $(a, b) \subset[0, \pi]$ such that $f(t)>0$ on $(a, b)$ and $f(a)=0$. In this situation, we observe

$$
\begin{aligned}
f^{\prime}(t) & =\frac{1}{\|X(t)-\bar{X}(t)\|}\left\langle X(t)-\bar{X}(t), X^{\prime}(t)-\bar{X}^{\prime}(t)\right\rangle \\
& \leq\left\|X^{\prime}(t)-\bar{X}^{\prime}(t)\right\|=\sqrt{\|\dot{c}(t)-\dot{\bar{c}}(t)\|^{2}+\|\ddot{c}(t)-\ddot{\bar{c}}(t)\|^{2}}
\end{aligned}
$$

on $(a, b)$. Since $\ddot{\bar{c}}(t)=-\bar{c}(t)$, we have

$$
\begin{aligned}
\|\ddot{c}(t)-\ddot{\bar{c}}(t)\| & =\|(\ddot{c}(t)+c(t))-(\ddot{\bar{c}}(t)+c(t))\| \\
& \leq\|\ddot{c}(t)+c(t)\|+\|\ddot{\bar{c}}(t)+c(t)\|=\|\ddot{c}(t)+c(t)\|+\|c(t)-\bar{c}(t)\| .
\end{aligned}
$$

Thus, by (3.4),

$$
\begin{aligned}
& \|\dot{c}(t)-\dot{\bar{c}}(t)\|^{2}+\|\ddot{c}(t)-\ddot{\bar{c}}(t)\|^{2} \\
& \leq\|\dot{c}(t)-\dot{\bar{c}}(t)\|^{2}+\|\ddot{c}(t)+c(t)\|^{2}+2\|\ddot{c}(t)+c(t)\| \cdot\|c(t)-\bar{c}(t)\|+\|c(t)-\bar{c}(t)\|^{2} \\
& \leq f^{2}(t)+2\|\ddot{c}(t)+c(t)\| f(t)+\|\ddot{c}(t)+c(t)\|^{2}=(f(t)+\|\ddot{c}(t)+c(t)\|)^{2} .
\end{aligned}
$$

Hence, by (3.3) and (3.5), we get

$$
f^{\prime}(t) \leq f(t)+\|\ddot{c}(t)+c(t)\|
$$

on $(a, b)$. Since $e^{-t}\left(f^{\prime}(t)-f(t)\right) \leq e^{-t}\|\ddot{c}(t)+c(t)\|$ from (3.6) , and since $f(a)=0$,

$$
\int_{a}^{b} e^{-t}\|\ddot{c}(t)+c(t)\| d t \geq \int_{a}^{b} e^{-t}\left(f^{\prime}(t)-f(t)\right) d t=\int_{a}^{b}\left(e^{-t} f(t)\right)^{\prime} d t=e^{-b} f(b),
$$

and hence $f(b) \leq e^{b} \int_{a}^{b} e^{-\theta}\|\ddot{c}(\theta)+c(\theta)\| d \theta \leq e^{b} \int_{0}^{b} e^{-\theta}\|\ddot{c}(\theta)+c(\theta)\| d \theta$. Since the function $t \longrightarrow e^{t} \int_{0}^{t} e^{-\theta}\|\ddot{c}(\theta)+c(\theta)\| d \theta$ is increasing on $[0, \pi]$,

$$
f(b) \leq e^{b} \int_{0}^{b} e^{-\theta}\|\ddot{c}(\theta)+c(\theta)\| d \theta \leq e^{\pi} \int_{0}^{\pi} e^{-\theta}\|\ddot{c}(\theta)+c(\theta)\| d \theta .
$$

If $f(t)=0$ for some $t \in[0, \pi]$, then (3.7) still holds for such a $t$. Therefore,

$$
f(t) \leq e^{\pi} \int_{0}^{\pi} e^{-\theta}\|\ddot{c}(\theta)+c(\theta)\| d \theta .
$$

holds on $[0, \pi]$. 
Lemma 3.3 $\langle c(0), c(\theta)\rangle \cos \theta \geq \cos ^{2} \theta-\alpha|\cos \theta|$ for all $\theta \in[0, \pi]$.

Proof. Since $\|c(0)\|=1$ and $c(0) \perp \dot{c}(0)$,

$$
\begin{aligned}
\langle c(0), c(\theta)\rangle & =\langle c(0), c(\theta)-\bar{c}(\theta)\rangle+\langle c(0), \bar{c}(\theta)\rangle \\
& =\langle c(0), c(\theta)-\bar{c}(\theta)\rangle+\langle c(0), c(0) \cos \theta+\dot{c}(0) \sin \theta\rangle \\
& =\langle c(0), c(\theta)-\bar{c}(\theta)\rangle+\cos \theta .
\end{aligned}
$$

By (3.2) and (3.8), we have

$$
\langle c(0), c(\theta)\rangle \cos \theta \geq \cos ^{2} \theta-\|c(\theta)-\bar{c}(\theta)\| \cdot|\cos \theta| \geq \cos ^{2} \theta-\alpha|\cos \theta| .
$$

Lemma $3.4\langle\dot{c}(0), c(\theta)\rangle \sin \theta \geq\|\dot{c}(0)\|^{2} \sin ^{2} \theta-\alpha\|\dot{c}(0)\| \sin \theta$ for all $\theta \in[0, \pi]$.

Proof. Since $c(0) \perp \dot{c}(0)$,

$$
\langle\dot{c}(0), c(\theta)\rangle=\langle\dot{c}(0), c(\theta)-\bar{c}(\theta)\rangle+\langle\dot{c}(0), \bar{c}(\theta)\rangle=\langle\dot{c}(0), c(\theta)-\bar{c}(\theta)\rangle+\|\dot{c}(0)\|^{2} \sin \theta .
$$

By (3.2) and (3.9), we have

$$
\begin{aligned}
\langle\dot{c}(0), c(\theta)\rangle \sin \theta & \geq\|\dot{c}(0)\|^{2} \sin ^{2} \theta-\|\dot{c}(0)\| \cdot\|c(\theta)-\bar{c}(\theta)\| \sin \theta \\
& \geq\|\dot{c}(0)\|^{2} \sin ^{2} \theta-\alpha\|\dot{c}(0)\| \sin \theta
\end{aligned}
$$

Lemma 3.5||$|\dot{c}(0) \|-1| \leq \alpha$.

Proof. Since $\bar{c}(\pi / 2)=\dot{c}(0), \dot{c}(0)=\bar{c}(\pi / 2)-c(\pi / 2)+c(\pi / 2)$. Since $\|c(\pi / 2)\|=1$, by the triangle inequality and (3.2), $\|\dot{c}(0)\| \leq\|\bar{c}(\pi / 2)-c(\pi / 2)\|+\|c(\pi / 2)\| \leq \alpha+1$, and $\|\dot{c}(0)\| \geq\|c(\pi / 2)\|-\|\bar{c}(\pi / 2)-c(\pi / 2)\| \geq 1-\alpha$. Hence, we get the assertion.

Lemma 3.6 For $\alpha=1-1 / \sqrt{2}$, we have

$$
\langle\bar{c}(\theta), c(\theta)\rangle>0
$$

on $[0, \pi]$.

Proof. First we consider the case where $\|\dot{c}(0)\| \geq 1$ holds. Then, by Lemmas 3.3, 3.4, and 3.5, we have

$$
\begin{aligned}
\langle\bar{c}(\theta), c(\theta)\rangle & =\langle c(0), c(\theta)\rangle \cos \theta+\langle\dot{c}(0), c(\theta)\rangle \sin \theta \\
& \geq \cos ^{2} \theta-\alpha|\cos \theta|+\|\dot{c}(0)\|^{2} \sin ^{2} \theta-\alpha\|\dot{c}(0)\| \sin \theta \\
& \geq \cos ^{2} \theta-\alpha|\cos \theta|+\sin ^{2} \theta-\alpha(\alpha+1) \sin \theta \\
& =1-\alpha|\cos \theta|-\alpha(\alpha+1) \sin \theta .
\end{aligned}
$$


Set $t=\sin \theta$. Note that $0 \leq t \leq 1$. Since $|\cos \theta|=\sqrt{1-t^{2}} \leq 1-t^{2} / 2$, we have, by (3.10),

$$
\begin{aligned}
\langle\bar{c}(\theta), c(\theta)\rangle & \geq 1-\alpha|\cos \theta|-\alpha(\alpha+1) \sin \theta \\
& \geq 1+\alpha\left(\frac{t^{2}}{2}-1\right)-\alpha(\alpha+1) t \\
& =\frac{\alpha}{2} t^{2}-\alpha(\alpha+1) t+1-\alpha \\
& =\frac{\alpha}{2}\{t-(\alpha+1)\}^{2}-\frac{\alpha}{2}(\alpha+1)^{2}+1-\alpha .
\end{aligned}
$$

Consider the function $\varphi(t):=(\alpha / 2) t^{2}-\alpha(\alpha+1) t+1-\alpha$ on $[0,1]$, which is a parabola. From (3.12), the axis of $\varphi$ is $t=\alpha+1$. Since $\alpha=1-1 / \sqrt{2}$, we see that $\alpha / 2>0$ and $\alpha+1>1$, which imply that $\varphi$ is decreasing on $[0,1]$. Moreover, since $\alpha=1-1 / \sqrt{2}$, we see that

$$
\varphi(1)=-\left(\alpha+\frac{3}{4}\right)^{2}+\frac{25}{16}>0 .
$$

Hence, by (3.11), (3.12), and (3.13),

$$
\langle\bar{c}(\theta), c(\theta)\rangle \geq \varphi(1)>0
$$

holds on $[0, \pi]$.

Finally we consider the case where $\|\dot{c}(0)\|<1$ holds. Then, since $\alpha=1-1 / \sqrt{2}$, we have, by Lemmas 3.3, 3.4, and 3.5,

$$
\begin{aligned}
\langle\bar{c}(\theta), c(\theta)\rangle & =\langle c(0), c(\theta)\rangle \cos \theta+\langle\dot{c}(0), c(\theta)\rangle \sin \theta \\
& \geq \cos ^{2} \theta-\alpha|\cos \theta|+\|\dot{c}(0)\|^{2} \sin ^{2} \theta-\alpha\|\dot{c}(0)\| \sin \theta \\
& >\cos ^{2} \theta+\sin ^{2} \theta-\alpha|\cos \theta|+\left(\|\dot{c}(0)\|^{2}-1\right) \sin ^{2} \theta-\alpha \sin \theta \\
& =1-\alpha(|\cos \theta|+\sin \theta)+(\|\dot{c}(0)\|+1)(\|\dot{c}(0)\|-1) \sin ^{2} \theta \\
& \geq 1-\alpha(|\cos \theta|+\sin \theta)-\alpha(\|\dot{c}(0)\|+1) \sin ^{2} \theta \\
& >1-\sqrt{2} \alpha-2 \alpha=0
\end{aligned}
$$

for all $\theta \in[0, \pi]$.

Lemma 3.7 Let $\widetilde{F}: \mathbb{R}^{n} \longrightarrow \mathbb{R}^{n}$ be the map defined by

$$
\widetilde{F}(v):= \begin{cases}\|v\| \sigma\left(\frac{v}{\|v\|}\right) & \text { on } \mathbb{R}^{n} \backslash\{o\} \\ o & \text { when } v=o .\end{cases}
$$

Then, the generalized differential of $\widetilde{F}$ at o is given by

$$
\partial \widetilde{F}(o)=\operatorname{Conv}\left(\left\{A_{v} \mid v \in S^{n-1}(1)\right\}\right),
$$

where $A_{v}$ is the linear map defined by $A_{v}(\lambda v):=\lambda \sigma(v)$ for all $\lambda \in \mathbb{R}$ and $A_{v}(w):=d \sigma_{v}(w)$ for all $w \in \mathbb{R}^{n}$ with $w \perp v$. 
Proof. Let $v \in S^{n-1}(1), \ell>0$, and $\lambda \in \mathbb{R}$. By (3.14),

$$
d \widetilde{F}_{\ell v}(\lambda v)=\left.\frac{d}{d t} \widetilde{F}(\ell v+t \lambda v)\right|_{t=0}=\left.\frac{d}{d t}(\ell+\lambda t) \sigma(v)\right|_{t=0}=\lambda \sigma(v) .
$$

For any $w \in \mathbb{R}^{n}$ with $w \perp v$, by (3.14),

$$
\begin{aligned}
d \widetilde{F}_{\ell v}(w)=\left.\frac{d}{d t} \widetilde{F}(\ell v+t w)\right|_{t=0} & =\left.\frac{d}{d t} \widetilde{F}\left(\sqrt{\ell^{2}+\|w\|^{2} t^{2}} \cdot \frac{\ell v+t w}{\sqrt{\ell^{2}+\|w\|^{2} t^{2}}}\right)\right|_{t=0} \\
& =\left.\frac{d}{d t} \sqrt{\ell^{2}+\|w\|^{2} t^{2}} \cdot \sigma\left(\frac{\ell v+t w}{\sqrt{\ell^{2}+\|w\|^{2} t^{2}}}\right)\right|_{t=0} \\
& =\left.\ell \cdot \frac{d}{d t} \sigma\left(\frac{\ell v+t w}{\sqrt{\ell^{2}+\|w\|^{2} t^{2}}}\right)\right|_{t=0} \\
& =\ell \cdot d \sigma_{v}\left(\frac{w}{\ell}\right)=d \sigma_{v}(w)
\end{aligned}
$$

Hence, by (3.15) and (3.16), we obtain $\partial \widetilde{F}(o)=\operatorname{Conv}\left(\left\{A_{v} \mid v \in S^{n-1}(1)\right\}\right)$.

Remark 3.8 In the proof of Lemma 3.7, we do not need the assumption that $\sigma$ satisfies (3.1) for some $\alpha$.

Now, we are going to give the proof of Theorem 3.1:

(Proof of Theorem 3.1) Let $\alpha>0$ be sufficiently small, and let $\widetilde{F}: \mathbb{R}^{n} \longrightarrow \mathbb{R}^{n}$ be the map defined by (3.14). Let $u, v \in S^{n-1}(1)$ be any vectors. Then, we can take a unit speed geodesic $\gamma:[0, \pi] \longrightarrow S^{n-1}(1)$ emanating from $v=\gamma(0)$ satisfying $u=\gamma\left(t_{0}\right)$ for some $t_{0} \in[0, \pi]$. Setting $w:=\dot{\gamma}(0)$, we have $\gamma(t)=v \cos t+w \sin t$ on $[0, \pi]$. Take any $\ell>0$, and fix it. Since $c(0)=\sigma(\gamma(0))=\sigma(v)$ and $\dot{c}(0)=d \sigma_{\gamma(0)}(\dot{\gamma}(0))=d \sigma_{v}(w)$, we see, by (3.15) and (3.16),

$$
\begin{aligned}
d \widetilde{F}_{\ell v}(u)=d \widetilde{F}_{\ell v}\left(\gamma\left(t_{0}\right)\right) & =d \widetilde{F}_{\ell v}(v) \cos t_{0}+d \widetilde{F}_{\ell v}(w) \sin t_{0} \\
& =\sigma(v) \cos t_{0}+d \sigma_{v}(w) \sin t_{0} \\
& =c(0) \cos t_{0}+\dot{c}(0) \sin t_{0}=\bar{c}\left(t_{0}\right) .
\end{aligned}
$$

Since $\sigma(u)=\sigma\left(\gamma\left(t_{0}\right)\right)=c\left(t_{0}\right)$,

$$
\left\langle d \widetilde{F}_{\ell v}(u), \sigma(u)\right\rangle=\left\langle d \widetilde{F}_{\ell v}(u), c\left(t_{0}\right)\right\rangle=\left\langle\bar{c}\left(t_{0}\right), c\left(t_{0}\right)\right\rangle .
$$

Thus, if $\alpha=1-1 / \sqrt{2}$, then, by Lemma 3.6, $\left\langle d \widetilde{F}_{\ell v}(u), \sigma(u)\right\rangle>0$ holds. This implies

$$
\left\langle A_{v}(u), \sigma(u)\right\rangle>0
$$

for all $u, v \in S^{n-1}(1)$. Therefore, by Lemma 3.7 and (3.18), $o$ is non-singular for $\widetilde{F}$. Indeed, suppose that $o$ is singular for $\widetilde{F}$. Set $G:=\left\{A_{v} \mid v \in S^{n-1}(1)\right\}$. Since

$$
\operatorname{Conv}(G)=\left\{\sum_{i=1}^{\ell} \lambda_{i} A_{z_{i}} \mid A_{z_{i}} \in G, \sum_{i=1}^{\ell} \lambda_{i}=1, \lambda_{i} \geq 0(i=1,2, \ldots, \ell)\right\},
$$


where $\ell<\infty$ (by Carathéodory's theorem), there exists $\sum_{i=1}^{\ell} \lambda_{i} A_{z_{i}} \in \partial \widetilde{F}(o)$ such that $\operatorname{rank}\left(\sum_{i=1}^{\ell} \lambda_{i} A_{z_{i}}\right)<n$. Thus, we may find a vector $v_{0} \in S^{n-1}(1)$ such that $\sum_{i=1}^{\ell} \lambda_{i} A_{z_{i}}\left(v_{0}\right)=$ o. By (3.18),

$$
0=\left\langle o, \sigma\left(v_{0}\right)\right\rangle=\left\langle\sum_{i=1}^{\ell} \lambda_{i} A_{z_{i}}\left(v_{0}\right), \sigma\left(v_{0}\right)\right\rangle=\sum_{i=1}^{\ell} \lambda_{i}\left\langle A_{z_{i}}\left(v_{0}\right), \sigma\left(v_{0}\right)\right\rangle>0,
$$

which is a contradiction. Hence, $o$ is non-singular for $\widetilde{F}$.

From (3.17) in the proof of Theorem 3.1, we have the following lemma, which is applied to the proof of the differentiable exotic sphere theorem II.

Lemma 3.9 If $\sigma$ satisfies

$$
\angle(\bar{c}(t), c(t))<\frac{\pi}{2}
$$

for all geodesic segments $\gamma([0, \pi]) \subset S^{n-1}(1)$ with $\|\dot{\gamma}\| \equiv 1$, then $o \in \mathbb{R}^{n}$ is non-singular for the map $\widetilde{F}$ defined by (3.14). Here, we do not assume that $\sigma$ satisfies (3.1) for some $\alpha$.

We need a corollary below of Theorem 3.1 to prove the differentiable exotic sphere theorem I in the case where (1.6) holds. We prepare the following lemma for proving the corollary.

Lemma 3.10 A smooth curve $c:(a, b) \longrightarrow S^{n-1}(1) \subset \mathbb{R}^{n}$ satisfies

$$
\|\ddot{c}(t)+c(t)\|^{2}=\|\ddot{c}(t)\|^{2}-2\|\dot{c}(t)\|^{2}+1 .
$$

Proof. It is clear that $\|\ddot{c}(t)+c(t)\|^{2}=\|\ddot{c}\|^{2}+2\langle\ddot{c}(t), c(t)\rangle+1$, where note that $\|c(t)\| \equiv 1$ on $(a, b)$. Since $1=\|c(t)\|^{2}=\langle c(t), c(t)\rangle$ for all $t \in(a, b),\langle\dot{c}(t), c(t)\rangle \equiv 0$ holds. We thus have $\langle\ddot{c}(t), c(t)\rangle=-\langle\dot{c}(t), \dot{c}(t)\rangle=-\|\dot{c}(t)\|^{2}$. Hence, we get

$$
\|\ddot{c}(t)+c(t)\|^{2}=\|\ddot{c}\|^{2}+2\langle\ddot{c}(t), c(t)\rangle+1=\|\ddot{c}(t)\|^{2}-2\|\dot{c}(t)\|^{2}+1 .
$$

Corollary 3.11 Let $\sigma: S^{n-1}(1) \longrightarrow S^{n-1}(1)$ be a diffeomorphism, $c:=\sigma \circ \gamma$ the curve in $S^{n-1}(1)$, where $\gamma:[0, \pi] \longrightarrow S^{n-1}(1)$ denotes a geodesic segment, and $\widetilde{F}: \mathbb{R}^{n} \longrightarrow \mathbb{R}^{n}$ the map defined by (3.14). If $\operatorname{Lip}^{\mathrm{b}}(\sigma)$ satisfies (1.6) for all geodesic segments $\gamma([0, \pi]) \subset$ $S^{n-1}(1)$ with $\|\dot{\gamma}\| \equiv 1$, then $o \in \mathbb{R}^{n}$ is non-singular for $\widetilde{F}$.

Proof. Since

$$
\|\dot{c}(t)\|=\lim _{h \rightarrow 0} \frac{\|c(t+h)-c(t)\|}{|h|}=\lim _{h \rightarrow 0} \frac{\|\sigma(\gamma(t+h))-\sigma(\gamma(t))\|}{\|\gamma(t+h)-\gamma(t)\|} \cdot \frac{\|\gamma(t+h)-\gamma(t)\|}{|h|},
$$

$$
\operatorname{Lip}^{\mathrm{b}}(\sigma)^{-1} \leq\|\dot{c}\| \leq \operatorname{Lip}^{\mathrm{b}}(\sigma)
$$


holds on any geodesic segment $\gamma([0, \pi]) \subset S^{n-1}(1)$. By Lemma 3.10, (1.6), and (3.19),

$$
\begin{aligned}
\int_{0}^{\pi} e^{-t}\|\ddot{c}(t)+c(t)\| d t & =\int_{0}^{\pi} e^{-t} \sqrt{\|\ddot{c}(t)\|^{2}-2\|\dot{c}(t)\|^{2}+1} d t \\
& \leq \int_{0}^{\pi} e^{-t} \sqrt{\|\ddot{c}(t)\|^{2}-2 \operatorname{Lip}^{\mathrm{b}}(\sigma)^{-2}+1} d t \\
& \leq \int_{0}^{\pi} e^{-t} d t \sqrt{2\left\{\frac{\sqrt{2}-1}{2\left(e^{\pi}-1\right)}\right\}^{2}} \\
& =\left(1-e^{-\pi}\right) \cdot \frac{2-\sqrt{2}}{2\left(e^{\pi}-1\right)} \\
& =e^{-\pi}\left(1-\frac{1}{\sqrt{2}}\right) .
\end{aligned}
$$

Hence, $\sigma$ satisfies (3.1) for $\alpha=1-1 / \sqrt{2}$. By Theorem 3.1, $o$ is non-singular for $\widetilde{F}$.

\subsection{Preliminary to the proof in assuming (1.7)}

Lemma 3.12 Let $\sigma: S^{n-1}(1) \longrightarrow S^{n-1}(1)$ be a diffeomorphism, where we do not assume (3.1), Lip ${ }^{\mathrm{b}}(\sigma)$ the bi-Lipschitz constant of it, and $\widetilde{F}: \mathbb{R}^{n} \longrightarrow \mathbb{R}^{n}$ the map defined by (3.14). Then, we have

$$
\operatorname{Lip}^{\mathrm{b}}(\sigma)^{-1}\|u-v\| \leq\|\widetilde{F}(u)-\widetilde{F}(v)\| \leq \operatorname{Lip}^{\mathrm{b}}(\sigma)\|u-v\|
$$

for all $u, v \in \mathbb{R}^{n}$, i.e., $\widetilde{F}$ is bi-Lipschitz.

Proof. Take any $u, v \in \mathbb{R}^{n}$. If $u=0$, or $v=0$, then the inequality holds. Hence, we assume $u \neq 0$ and $v \neq 0$. Set $\tilde{u}:=u /\|u\|$ and $\tilde{v}:=v /\|v\|$. Note that if $\tilde{u}=\tilde{v}$, then (3.20) trivially holds. Hence, we assume $\tilde{u} \neq \tilde{v}$. Since $\|\widetilde{F}(u)\|=\|u\|$ and $\langle\widetilde{F}(u), \widetilde{F}(v)\rangle=$ $\|u\| \cdot\|v\|\langle\sigma(\tilde{u}), \sigma(\tilde{v})\rangle$,

$$
\|\widetilde{F}(u)-\widetilde{F}(v)\|^{2}=\|u\|^{2}+\|v\|^{2}-2\|u\| \cdot\|v\|\langle\sigma(\tilde{u}), \sigma(\tilde{v})\rangle
$$

Since $\|u-v\|^{2}=\|u\|^{2}+\|v\|^{2}-2\|u\| \cdot\|v\|\langle\tilde{u}, \tilde{v}\rangle$, we have, by (3.21),

$$
\|\widetilde{F}(u)-\widetilde{F}(v)\|^{2}-\|u-v\|^{2}=2\|u\| \cdot\|v\|(\langle\tilde{u}, \tilde{v}\rangle-\langle\sigma(\tilde{u}), \sigma(\tilde{v})\rangle)
$$

Similarly, we see

$$
\|\sigma(\tilde{u})-\sigma(\tilde{v})\|^{2}-\|\tilde{u}-\tilde{v}\|^{2}=2(\langle\tilde{u}, \tilde{v}\rangle-\langle\sigma(\tilde{u}), \sigma(\tilde{v})\rangle)
$$

Set $\ell^{2}:=\|\sigma(\tilde{u})-\sigma(\tilde{v})\|^{2} /\|\tilde{u}-\tilde{v}\|^{2}$. Then, by (3.22) and (3.23),

$$
\begin{aligned}
\|\widetilde{F}(u)-\widetilde{F}(v)\|^{2}-\|u-v\|^{2} & =\|u\| \cdot\|v\|\left\{\|\sigma(\tilde{u})-\sigma(\tilde{v})\|^{2}-\|\tilde{u}-\tilde{v}\|^{2}\right\} \\
& =\|u\| \cdot\|v\|\left(\ell^{2}-1\right)\|\tilde{u}-\tilde{v}\|^{2} \\
& =2\left(\ell^{2}-1\right)(\|u\| \cdot\|v\|-\langle u, v\rangle) .
\end{aligned}
$$


Since $\operatorname{Lip}^{\mathrm{b}}(\sigma) \geq 1,\|u\| \cdot\|v\|-\langle u, v\rangle \geq 0$ and $2(\|u\| \cdot\|v\|-\langle u, v\rangle) \leq\|u-v\|^{2}$, we see, by (3.24),

$$
\begin{aligned}
\|\widetilde{F}(u)-\widetilde{F}(v)\|^{2} & =\|u-v\|^{2}+2\left(\ell^{2}-1\right)(\|u\| \cdot\|v\|-\langle u, v\rangle) \\
& \leq\|u-v\|^{2}+2\left(\operatorname{Lip}^{\mathrm{b}}(\sigma)^{2}-1\right)(\|u\| \cdot\|v\|-\langle u, v\rangle) \\
& =\operatorname{Lip}^{\mathrm{b}}(\sigma)^{2}\|u-v\|^{2}+\left(1-\operatorname{Lip}^{\mathrm{b}}(\sigma)^{2}\right)\left\{\|u-v\|^{2}-2(\|u\| \cdot\|v\|-\langle u, v\rangle)\right\} \\
& \leq \operatorname{Lip}^{\mathrm{b}}(\sigma)^{2}\|u-v\|^{2} .
\end{aligned}
$$

As well as above, we have $\|\widetilde{F}(u)-\widetilde{F}(v)\|^{2} \geq \operatorname{Lip}^{\mathrm{b}}(\sigma)^{-2}\|u-v\|^{2}$.

\subsection{The proofs}

All notations in this subsection are the same as those defined in Sect.1.3.

(Proof of the differentiable exotic sphere theorem I in the case of (1.6)) We assume here $d_{M_{i}}\left(p_{i}, q_{i}\right):=\pi$ for each $i=1,2$ by normalizing the metric, where $d_{M_{i}}$ denotes the distance function on $M_{i}$. Note that this normalization does not change $\operatorname{Lip}^{\mathrm{b}}(\sigma)$ and $\|\ddot{c}\|$. The diffeomorphism $\sigma_{q_{i}}^{p_{i}}: \mathbb{S}_{p_{i}}^{n-1} \longrightarrow \mathbb{S}_{q_{i}}^{n-1}$ defined by (1.2) satisfies $\sigma_{q_{i}}^{p_{i}}\left(u_{i}\right)=-\dot{\tau}_{u_{i}}(\pi)$, i.e., $\ell_{i}=\pi$, since $d_{M_{i}}\left(p_{i}, q_{i}\right)=\pi$. Here, $\tau_{u_{i}}(t):=\exp _{p_{i}} t u_{i}$ for all $u_{i} \in \mathbb{S}_{p_{i}}^{n-1}$ and all $t \in[0, \pi]$.

We first construct a map $\widetilde{F}$ satisfying (3.14) under identifying $T_{q_{i}} M_{i}=\mathbb{R}^{n}$ : For each $\left(t, u_{1}\right) \in[0, \pi] \times \mathbb{S}_{p_{1}}^{n-1}$, we define the bi-Lipschitz homeomorphism $F: M_{1} \longrightarrow M_{2}$ by

$$
F\left(\exp _{p_{1}} t u_{1}\right):=\exp _{p_{2}}\left(t I\left(u_{1}\right)\right),
$$

where $I: T_{p_{1}} M_{1} \longrightarrow T_{p_{2}} M_{2}$ denotes a linear isometry. Note that $F$ is a diffeomorphism between $M_{1} \backslash\left\{q_{1}\right\}$ and $M_{2} \backslash\left\{q_{2}\right\}$. Define the map $\widetilde{F}: \mathbb{B}_{\pi}\left(o_{q_{1}}\right) \longrightarrow \mathbb{B}_{\pi}\left(o_{q_{2}}\right)$ by

$$
\widetilde{F}:=\exp _{q_{2}}^{-1} \circ F \circ \exp _{q_{1}},
$$

where for each $i=1,2, \mathbb{B}_{\pi}\left(o_{q_{i}}\right):=\left\{v \in T_{q_{i}} M_{i} \mid\|v\|<\pi\right\}$ and $o_{q_{i}}$ denotes the origin of $T_{q_{i}} M_{i}$. Since

$$
F\left(\exp _{q_{1}} t \sigma_{q_{1}}^{p_{1}}\left(u_{1}\right)\right)=F\left(\tau_{u_{1}}(\pi-t)\right)=\exp _{p_{2}}(\pi-t) I\left(u_{1}\right)=\tau_{u_{2}}(\pi-t)=\exp _{q_{2}} t \sigma_{q_{2}}^{p_{2}}\left(u_{2}\right),
$$

where $u_{2}:=I\left(u_{1}\right)$, we have

$$
\widetilde{F}\left(t \sigma_{q_{1}}^{p_{1}}\left(u_{1}\right)\right)=\exp _{q_{2}}^{-1}\left(\exp _{q_{2}} t \sigma_{q_{2}}^{p_{2}}\left(u_{2}\right)\right)=t \sigma_{q_{2}}^{p_{2}}\left(u_{2}\right)=t \sigma_{q_{2}}^{p_{2}} \circ I\left(u_{1}\right)
$$

By setting $v_{1}:=\sigma_{q_{1}}^{p_{1}}\left(u_{1}\right)$, it follows from (3.27) that

$$
\widetilde{F}\left(t v_{1}\right)=\widetilde{F}\left(t \sigma_{q_{1}}^{p_{1}}\left(u_{1}\right)\right)=t \sigma_{q_{2}}^{p_{2}} \circ I\left(u_{1}\right)=t \sigma_{q_{2}}^{p_{2}} \circ I \circ \sigma_{p_{1}}^{q_{1}}\left(v_{1}\right)=t \sigma\left(v_{1}\right)
$$

and hence $\widetilde{F}$ satisfies (3.14).

Since $d\left(\exp _{q_{1}}\right)_{o}$ and $d\left(\exp _{q_{2}}^{-1}\right)_{q_{2}}$ are the identity maps, $\partial F\left(q_{1}\right)=\partial \widetilde{F}(o)$, where $o:=o_{q_{1}}$. By Lemma 3.7, $\partial F\left(q_{1}\right)=\operatorname{Conv}\left(\left\{A_{v} \mid v \in \mathbb{S}_{q_{1}}^{n-1}\right\}\right)$ holds, where $A_{v}$ is as in the statement. 
Since $\operatorname{Lip}^{\mathrm{b}}(\sigma)$ satisfies (1.6), by Corollary $3.11, q_{1}$ is non-singular for $F$. Since $F$ has no singular points on $M_{1}$, it follows from Theorem 1.3 that for any sufficiently small $\eta>0$, there exists a smooth approximation $f_{\eta}: M_{1} \longrightarrow M_{2}$ of $F$, which is an immersion. Since $f_{\eta}\left(M_{1}\right)$ is open and closed in $M_{2}, f_{\eta}\left(M_{1}\right)=M_{2}$. On the other hand, since $M_{1}$ is compact and $M_{2}$ is Hausdorff, $f_{\eta}$ is proper. Since $f_{\eta}$ is a local diffeomorphism, $f_{\eta}$ is a covering map from $M_{1}$ onto $M_{2}$. Since $M_{2}$ is simply connected, $f_{\eta}$ is injective. Therefore, for any sufficiently small $\eta>0, f_{\eta}$ is a diffeomorphism from $M_{1}$ onto $M_{2}$.

(Proof of the differentiable exotic sphere theorem I in the case of (1.7)) Let $F: M_{1} \longrightarrow M_{2}$ and $\widetilde{F}: \mathbb{B}_{\pi}\left(o_{q_{1}}\right) \longrightarrow \mathbb{B}_{\pi}\left(o_{q_{2}}\right)$ be the maps defined by (3.25) and (3.26), respectively. Moreover, let $M_{2}$ be isometrically embedded into $\mathbb{R}^{m}$, where $m \geq n+1$, by introducing the induced metric from the space. Then, $F: M_{1} \longrightarrow \mathbb{R}^{m}$. By Lemma 3.12 and (1.7), we get $\operatorname{Lip}^{\mathrm{b}}(\widetilde{F})^{2} \leq 1+\{(8 / \pi)(n-1)\}^{-1 / 2}$. Hence, it follows from the proof of [21, Theorem 5.1] that the smooth approximation $\widetilde{F}_{\varepsilon}$, which is the convolution of $\widetilde{F}$ and the mollifier $\rho_{\varepsilon}$, is an immersion from some open ball $\mathbb{B}_{\delta}\left(o_{q_{1}}\right) \subset \mathbb{B}_{\pi}\left(o_{q_{1}}\right)$ into $\mathbb{B}_{\pi}\left(o_{q_{2}}\right)$. Here, $\widetilde{F}_{\varepsilon}(y):=\int \widetilde{F}(x) \rho_{\varepsilon}(x-y) d x$ for $\varepsilon<\delta$. Therefore, $F$ admits a local smooth approximation $F_{\varepsilon}^{\left(q_{1}\right)}$ from an open ball $B_{\delta}\left(q_{1}\right)=\exp _{q_{1}} \mathbb{B}_{\delta}\left(o_{q_{1}}\right)$ into $\mathbb{R}^{m}$, which is an immersion. Let $\varphi: M_{1} \longrightarrow \mathbb{R}$ be a smooth function satisfying $0 \leq \varphi \leq 1$ on $M_{1}, \varphi \equiv 1$ on $\overline{B_{r}\left(q_{1}\right)}$, and $\operatorname{supp} \varphi \subset B_{R}\left(q_{1}\right)$, where $0<r<R<\delta$. Define the map $F_{\varepsilon}: M_{1} \longrightarrow \mathbb{R}^{m}$ by $F_{\varepsilon}:=(1-\varphi) F+\varphi F_{\varepsilon}^{\left(q_{1}\right)}$. Since

$$
F_{\varepsilon}= \begin{cases}F_{\varepsilon}^{\left(q_{1}\right)} & \text { on } \overline{B_{r}\left(q_{1}\right)}, \\ F & \text { on } M_{1} \backslash \operatorname{supp} \varphi\end{cases}
$$

$F_{\varepsilon}$ is a local diffeomorphism from $B_{r}\left(q_{1}\right) \cup\left(M_{1} \backslash \operatorname{supp} \varphi\right)$ into $\mathbb{R}^{m}$. Since $F$ is smooth on $B_{R}\left(q_{1}\right) \backslash \overline{B_{r}\left(q_{1}\right)}$, it is easy to see that $F_{\varepsilon}^{\left(q_{1}\right)}$ uniformly converges to $F$ on $\overline{B_{R}\left(q_{1}\right)} \backslash B_{r}\left(q_{1}\right)$ as $\varepsilon$ goes to zero in the $C^{1}$-topology. Thus, $F_{\varepsilon}$ uniformly converges to $F$ on $\overline{B_{R}\left(q_{1}\right)} \backslash B_{r}\left(q_{1}\right)$ as $\varepsilon$ goes to zero in the $C^{1}$-topology. Now, choose a small neighborhood $\mathcal{N}_{M_{2}}$ of $M_{2} \subset \mathbb{R}^{m}$ such that any $x \in \mathcal{N}_{M_{2}}$ admits a unique nearest point $\pi_{M_{2}}(x) \in M_{2}$. Then, the map $\pi_{M_{2}}: \mathcal{N}_{M_{2}} \longrightarrow M_{2}$ is well-defined and smooth. Since $\left(d \pi_{M_{2}}\right)_{y}$ is an orthogonal projection to $T_{y} M_{2}$ for each $y \in M_{2}$, the map $G_{\varepsilon}:=\pi_{M_{2}} \circ F_{\varepsilon}: M_{1} \longrightarrow M_{2}$ is an immersion for any sufficiently small $\varepsilon>0$. Since $G_{\varepsilon}\left(M_{1}\right)$ is open and closed in $M_{2}, G_{\varepsilon}\left(M_{1}\right)=M_{2}$. Moreover, since $M_{1}$ is compact and $M_{2}$ is Hausdorff, $G_{\varepsilon}$ is proper, and thus $G_{\varepsilon}$ is a covering map from $M_{1}$ onto $M_{2}$. Since $M_{2}$ is simply connected, $G_{\varepsilon}$ is injective, and hence it is a diffeomorphism from $M_{1}$ onto $M_{2}$ for any sufficiently small $\varepsilon>0$.

(Proof of the differentiable exotic sphere theorem II) Let $F: M_{1} \longrightarrow M_{2}$ and $\widetilde{F}$ : $\mathbb{B}_{\pi}\left(o_{q_{1}}\right) \longrightarrow \mathbb{B}_{\pi}\left(o_{q_{2}}\right)$ be the maps defined by (3.25) and (3.26), respectively. By the same argument in the proof of the case where (1.6) holds, we have $\partial F\left(q_{1}\right)=\partial \widetilde{F}(o)=$ $\operatorname{Conv}\left(\left\{A_{v} \mid v \in \mathbb{S}_{q_{1}}^{n-1}\right\}\right)$, where $A_{v}$ is as in Lemma 3.7. Since $\sigma$ satisfies (1.10), by Lemma 3.9, $q_{1}$ is non-singular for $F$, i.e., $F$ has no singular points on $M_{1}$. By Theorem 1.3 and the same argument as the proof of the case where (1.6) holds, $M_{1}$ and $M_{2}$ are diffeomorphic. 


\subsection{The reason why we need the restriction of $\ddot{c}$ in the condition (1.6)}

Let $\sigma: S^{n-1}(1) \longrightarrow S^{n-1}(1)$ be a diffeomorphism, where $S^{n-1}(1):=\left\{v \in \mathbb{R}^{n} \mid\|v\|=1\right\}$, and let $\operatorname{Lip}^{\mathrm{b}}(\sigma) \geq 1$ denote the bi-Lipschitz constant of $\sigma$ defined by (1.4). First, we will prove that, for any $i, j \in\{1,2, \ldots, n\}$,

$$
\left|\left\langle\sigma\left(e_{i}\right), \sigma\left(e_{j}\right)\right\rangle-\delta_{i j}\right| \leq \operatorname{Lip}^{\mathrm{b}}(\sigma)^{2}-1,
$$

where $e_{i}:=(0, \ldots, 1, \ldots, 0) \in \mathbb{R}^{n}$. From this property, $\left\{\sigma\left(e_{1}\right), \sigma\left(e_{2}\right), \ldots, \sigma\left(e_{n}\right)\right\}$ looks linearly independent if $\operatorname{Lip}^{\mathrm{b}}(\sigma)^{2}-1$ is sufficiently small. As Lemma 3.13 below shows, however, $\left\{\sigma\left(e_{1}\right), \sigma\left(e_{2}\right), \ldots, \sigma\left(e_{n}\right)\right\}$ is not always linearly independent. Take any vectors $u, v \in S^{n-1}(1)$. By the parallelogram law, we have

$$
\langle\sigma(u), \sigma(v)\rangle=\frac{1}{2}\left\{\|\sigma(u)\|^{2}+\|\sigma(v)\|^{2}-\|\sigma(u)-\sigma(v)\|^{2}\right\}=\frac{1}{2}\left\{2-\|\sigma(u)-\sigma(v)\|^{2}\right\}
$$

and $\langle u, v\rangle=\left\{\|u\|^{2}+\|v\|^{2}-\|u-v\|^{2}\right\} / 2=\left\{2-\|u-v\|^{2}\right\} / 2$. Then, we have

$$
\begin{aligned}
\langle\sigma(u), \sigma(v)\rangle-\langle u, v\rangle & =\frac{1}{2}\left\{\|u-v\|^{2}-\|\sigma(u)-\sigma(v)\|^{2}\right\} \\
& \leq \frac{1}{2}\left(1-\operatorname{Lip}^{\mathrm{b}}(\sigma)^{-2}\right)\|u-v\|^{2} \leq \frac{\operatorname{Lip}^{\mathrm{b}}(\sigma)^{2}-1}{2}\|u-v\|^{2} .
\end{aligned}
$$

As well as above, $\langle\sigma(u), \sigma(v)\rangle-\langle u, v\rangle \geq\left(1-\operatorname{Lip}^{\mathrm{b}}(\sigma)^{2}\right)\|u-v\|^{2} / 2$. Thus,

$$
|\langle\sigma(u), \sigma(v)\rangle-\langle u, v\rangle| \leq \frac{\operatorname{Lip}^{\mathrm{b}}(\sigma)^{2}-1}{2}\|u-v\|^{2}
$$

holds. In particular, $\left|\left\langle\sigma\left(e_{i}\right), \sigma\left(e_{j}\right)\right\rangle-\left\langle e_{i}, e_{j}\right\rangle\right| \leq \operatorname{Lip}^{\mathrm{b}}(\sigma)^{2}-1$.

Lemma 3.13 For any $\varepsilon>0$, there exist $\boldsymbol{a}_{1}, \boldsymbol{a}_{2}, \ldots, \boldsymbol{a}_{n} \in \mathbb{R}^{n}$ such that

$$
\left|\left\langle\boldsymbol{a}_{i}, \boldsymbol{a}_{j}\right\rangle-\delta_{i j}\right|<\varepsilon
$$

but $\left\{\boldsymbol{a}_{1}, \boldsymbol{a}_{2}, \ldots, \boldsymbol{a}_{n}\right\}$ is not linearly independent.

Proof. Take any $\varepsilon \in(0,1)$, and fix it. Choose an integer $k \geq 0$ satisfying

$$
\frac{4\left(1-\varepsilon^{2}\right)}{\varepsilon^{2}}<k \leq \frac{4\left(1-\varepsilon^{2}\right)}{\varepsilon^{2}}+1 .
$$

Set $c_{1}=c_{2}=\cdots=c_{k}=\varepsilon / 2$. Since $\sum_{j=1}^{k} c_{j}^{2}=k \varepsilon^{2} / 4$, we have $1-\sum_{j=1}^{k} c_{j}^{2}=1-k \varepsilon^{2} / 4$. By the right inequality of (3.29),

$$
1-\sum_{j=1}^{k} c_{j}^{2} \geq 1-\frac{\varepsilon^{2}}{4}\left\{\frac{4\left(1-\varepsilon^{2}\right)}{\varepsilon^{2}}+1\right\}=\frac{3}{4} \varepsilon^{2}>0 .
$$


By the left inequality of (3.29),

$$
1-\sum_{j=1}^{k} c_{j}^{2}<1-\frac{\varepsilon^{2}}{4} \cdot \frac{4\left(1-\varepsilon^{2}\right)}{\varepsilon^{2}}=\varepsilon^{2} .
$$

Thus, we have $0<c_{k+1}:=\sqrt{1-\sum_{j=1}^{k} c_{j}^{2}}<\varepsilon$. Defining $\boldsymbol{a}_{1}:=e_{1}, \boldsymbol{a}_{2}:=e_{2}, \ldots, \boldsymbol{a}_{k}:=$ $e_{k}, \boldsymbol{a}_{k+1}=\sum_{j=1}^{k+1} c_{j} e_{j} \in \mathbb{R}^{n}$, we see $\left\langle\boldsymbol{a}_{i}, \boldsymbol{a}_{j}\right\rangle=\delta_{i j}$ for all $i, j<k+1,\left\langle\boldsymbol{a}_{k+1}, \boldsymbol{a}_{k+1}\right\rangle=1$, and $0<\left\langle\boldsymbol{a}_{k+1}, \boldsymbol{a}_{i}\right\rangle<\varepsilon$ for all $i \neq k+1$. Hence, $\left\{\boldsymbol{a}_{1}, \boldsymbol{a}_{2}, \ldots, \boldsymbol{a}_{k+1}\right\}$ is linearly dependent, but that satisfies (3.28).

Remark 3.14 By Lemma 3.13, it looks impossible to find a bound on the Lipschitz constant of $\widetilde{F}$ independent of the dimension $n$ such that the differential of the smooth approximation of $\widetilde{F}$ at $o$ is injective. In this sense, it is natural that the Lipschitz constant of the locally bi-Lipschitz map in [21, Theorem 5.1] depends on $n$, and it is too for (1.7).

\section{Proof of the differentiable twisted sphere theorem}

We need two lemmas in order to prove the differentiable twisted sphere theorem.

Lemma 4.1 Let $M$ be a complete Riemannian manifold, and let $f: M \longrightarrow \mathbb{R}$ be a Lipschitz function on $M$. If $f^{-1}(0)$ is compact, then for any open neighborhood $U$ of $f^{-1}(0)$, there exists a positive number $\varepsilon_{0}$ such that for all $\varepsilon \in\left(0, \varepsilon_{0}\right), f_{\varepsilon}^{-1}(0) \subset U$, where $f_{\varepsilon}$ denotes the smooth approximation of $f$ defined in Sect.2.1.

Proof. For each $\varepsilon>0$, let $f_{\varepsilon}$ be the smooth approximation of the function $f$, i.e., $f_{\varepsilon}(q):=\sum_{i} \psi_{i}(q) f_{\varepsilon}^{\left(p_{i}\right)}(q)$. Here, $\left\{\psi_{i}\right\}_{i=1}^{\ell}$ denotes the partition of unity subordinate to $\left\{B_{r_{i} / 2}\left(p_{i}\right)\right\}$, where $\left\{B_{r_{i}}\left(p_{i}\right)\right\}$ is a locally finite covering of strongly convex balls of $M$ which satisfies $M=\cup_{i} B_{r_{i} / 2}\left(p_{i}\right)$, and the local approximation $f_{\varepsilon}^{\left(p_{i}\right)}$ is defined by the equation (2.1). Since $\sum_{i=1}^{\ell} \psi_{i}(q)=1$, we have, by the triangle inequality,

$$
\left|f_{\varepsilon}(q)-f(q)\right|=\left|\sum_{i=1}^{\ell} \psi_{i}(q)\left(f_{\varepsilon}^{\left(p_{i}\right)}(q)-f(q)\right)\right| \leq \sum_{i=1}^{\ell} \psi_{i}(q)\left|f_{\varepsilon}^{\left(p_{i}\right)}(q)-f(q)\right| .
$$

Applying Lemma 2.2 to (4.1), we see that

$$
\left|f_{\varepsilon}(q)-f(q)\right| \leq \varepsilon \cdot \operatorname{Lip}(f) \sum_{i=1}^{\ell} \psi_{i}(q) \operatorname{Lip}\left(\exp _{p_{i}} \mid \mathbb{B}_{r_{i}}\left(o_{p_{i}}\right)\right)
$$

for all $\varepsilon \in\left(0, \varepsilon_{0}\right)$, where $\varepsilon_{0}:=\min \left\{\varepsilon_{i} \mid i=1,2, \ldots, \ell\right\}$. Hence, for any sufficiently small $\varepsilon>0, f_{\varepsilon}^{-1}(0)$ is a subset of $U$.

Lemma 4.2 Let $A, B$ be linear transformations on $\mathbb{R}^{n}$ such that $\left.A\right|_{\mathbb{R}^{n-1}}=\left.B\right|_{\mathbb{R}^{n-1}}=$ $\operatorname{id}_{\mathbb{R}^{n-1}}$, and that $\langle A \vec{n}, \vec{n}\rangle>0,\langle B \vec{n}, \vec{n}\rangle>0$, where $\mathbb{R}^{n-1}:=\left\{\left(x_{1}, x_{2}, \ldots, x_{n}\right) \in \mathbb{R}^{n} \mid x_{n}=\right.$ $0\}$ and $\vec{n}:=(0, \ldots, 0,1)$. Then, every element in $\operatorname{Conv}(\{A, B\})$ is of maximal rank. 
Proof. Take any $\lambda \in[0,1]$. Assume that there exists $\vec{v}+a \vec{n} \in \mathbb{R}^{n}$, where $a \in \mathbb{R}$ and $\vec{v} \in \mathbb{R}^{n-1}$, such that $(\lambda A+(1-\lambda) B)(\vec{v}+a \vec{n})=o$. Since $o=(\lambda A+(1-\lambda) B)(\vec{v}+a \vec{n})=$ $\vec{v}+a(\lambda A+(1-\lambda) B) \vec{n}$, we have

$$
0=\langle\vec{v}+a(\lambda A+(1-\lambda) B) \vec{n}, \vec{n}\rangle=a(\lambda\langle A \vec{n}, \vec{n}\rangle+(1-\lambda)\langle B \vec{n}, \vec{n}\rangle),
$$

and hence $a=0$. Since $(\lambda A+(1-\lambda) B)(\vec{v}+a \vec{n})=o$, we have $\vec{v}=o$. Thus, $\vec{v}+a \vec{n}=o$. This implies that $\lambda A+(1-\lambda) B$ is non-singular for any $\lambda \in[0,1]$.

In what follows, all notations are the same as those defined in Sect.1.3.

(Proof of the differentiable twisted sphere theorem) We first prove (T-1), i.e., $M$ is a twisted sphere: Let $d_{p}, d_{q}$ be the distance functions from $p, q$, respectively, i.e., $d_{p}(x):=d(p, x)$ for all $x \in M$. Consider the Lipschitz function $f:=d_{p}-d_{q}$ on $M$. Remark that $f^{-1}(0)=$ $E_{p, q}$ is compact. Let $f_{\varepsilon}$ denote the smooth approximation of $f$, i.e., $f_{\varepsilon}=\left(d_{p}\right)_{\varepsilon}-\left(d_{q}\right)_{\varepsilon}$. Applying Lemma 2.14 to the compact set $K=E_{p, q}$, we obtain that $\nabla f_{\varepsilon} \neq 0$ on $E_{p, q}$ for all sufficiently small $\varepsilon>0$. Choose any $R>0$ so as to be $p, q \notin B_{R}\left(f^{-1}(0)\right)$. Here $B_{R}\left(f^{-1}(0)\right):=\left\{x \in M \mid \widetilde{d}\left(f^{-1}(0), x\right)<R\right\}$, where $\widetilde{d}\left(f^{-1}(0), x\right)=\min _{y \in f^{-1}(0)} d(y, x)$. By Lemma 4.1, $f_{\varepsilon}^{-1}(0) \subset B_{R}\left(f^{-1}(0)\right)$ for any sufficiently small $\varepsilon>0$, and hence $f_{\varepsilon}^{-1}(0)$ is a regular compact hypersurface. Choose such a sufficiently small $\varepsilon$, and fix it in the following. Now, let $\overline{D_{\varepsilon}(p)}:=\left\{x \in M \mid f_{\varepsilon}(x) \leq 0\right\}$ and $\overline{D_{\varepsilon}(q)}:=\left\{x \in M \mid f_{\varepsilon}(x) \geq 0\right\}$. Since $d_{p}$ is smooth on a punctured convex ball $\mathcal{P}(p)$ at $p,\left\|\nabla\left(d_{p}\right)_{\varepsilon}-\nabla d_{p}\right\|$ is sufficiently small on $\mathcal{P}(p)$. Therefore, we can assume that there exists a non-zero smooth vector field $X_{+}$on $\overline{D_{\varepsilon}(p)} \backslash\{p\}$ such that

$$
X_{+}= \begin{cases}\nabla d_{p} & \text { on } \mathcal{P}(p), \\ \nabla\left(d_{p}\right)_{\varepsilon} & \text { on a neighborhood of } f_{\varepsilon}^{-1}(0) .\end{cases}
$$

For each $v \in \mathbb{S}_{p}^{n-1}$, let $\tau_{+}(t, v)$ be the integral curve of $X_{+}$with initial conditions $\tau_{+}(0, v)=$ $p$ and $\frac{\partial \tau_{+}}{\partial t}(0, v)=v$. Since $X_{+}=\nabla d_{p}$ on $\mathcal{P}(p), \tau_{+}(t, v)=\exp _{p} t v$ holds for all sufficiently small $t \geq 0$. It follows from Lemma 2.14 that $\angle\left(\nabla\left(d_{p}\right)_{\varepsilon}, \nabla\left(d_{q}\right)_{\varepsilon}\right)>\pi / 2$ on $E_{p, q}$. Thus, there exists a smooth solution $t=t_{+}(v)>0$ of $f_{\varepsilon}\left(\tau_{+}(t, v)\right)=0$, since $X_{+}$is not tangent to $f_{\varepsilon}^{-1}(0)$ at each point of the hypersurface. Then, we have the diffeomorphism $G_{+}$from $\overline{D_{\varepsilon}(p)}$ onto $S_{+}^{n}(1):=\left\{\left(x_{1}, x_{2}, \ldots, x_{n+1}\right) \in S^{n}(1) \mid x_{n+1} \geq 0\right\}$ defined by

$$
G_{+}\left(\tau_{+}(t, v)\right):=\exp _{N} \frac{t \pi}{2 t_{+}(v)} I(v)
$$

for all $(t, v) \in[0, \infty) \times \mathbb{S}_{p}^{n-1}$ with $t \leq t_{+}(v)$, where $I$ denotes a linear isometry from $T_{p} M$ onto $T_{N} S^{n}(1)$. In the same way as $G_{+}$, we have the diffeomorphism $G_{-}$from $\overline{D_{\varepsilon}(q)}$ onto $S_{-}^{n}(1):=\left\{\left(x_{1}, x_{2}, \ldots, x_{n+1}\right) \in S^{n}(1) \mid x_{n+1} \leq 0\right\}$. Thus, we can define the induced metrics $g_{ \pm}:=G_{ \pm}^{*} g_{0}$ on $\overline{D_{\varepsilon}(p)}, \overline{D_{\varepsilon}(q)}$ from $S^{n}(1)$, where $g_{0}$ denotes the metric of $S^{n}(1)$, so that $\overline{D_{\varepsilon}(p)}, \overline{D_{\varepsilon}(q)}$ are isometric to $S_{+}^{n}(1), S_{-}^{n}(1)$, respectively. Let $\exp ^{g_{+}}$, $\exp ^{g_{-}}$be exponential maps on tangent spaces at $p, q$ of $\overline{D_{\varepsilon}(p)}, \overline{D_{\varepsilon}(q)}$ with respect to $g_{ \pm}$, respectively. For each $(t, v) \in[0, \pi] \times \mathbb{S}_{p}^{n-1}$, we define the point $e(t, v)$ on $M$ with respect to $g_{ \pm}$by

$$
e(t, v)= \begin{cases}\exp ^{g_{+}}(t v) & \text { on }[0, \pi / 2], \\ \exp ^{g_{-}}\left((\pi-t) \sigma_{q}^{p}(v)\right) & \text { on }[\pi / 2, \pi]\end{cases}
$$


where $\sigma_{q}^{p}: \mathbb{S}_{p}^{n-1} \longrightarrow \mathbb{S}_{q}^{n-1}$ is the diffeomorphism satisfying $\exp ^{+}(\pi v / 2)=\exp ^{-}\left(\pi \sigma_{q}^{p}(v) / 2\right)$. Thus, we have a boundary diffeomorphism $h_{\sigma_{q}^{p}}: \partial S_{+}^{n}(1) \longrightarrow \partial S_{-}^{n}(1)$ induced from $\sigma_{q}^{p}$. Hence, $M=S_{+}^{n}(1) \cup_{\sigma_{q}^{p}} S_{-}^{n}(1)$ is twisted.

We next prove (T-2), i.e., we construct a bi-Lipschitz homeomorphism from $M$ to $S^{n}(1)$ that admits a diffeomorphism between $M \backslash\{q\}$ and $S^{n}(1) \backslash\{S\}$ : For every $(t, v) \in$ $[0, \pi] \times \mathbb{S}_{p}^{n-1}$, define the map $F: M \longrightarrow S^{n}(1)$ by

$$
F(e(t, v)):=\exp _{N} t I(v) .
$$

It is not difficult to see that $F$ is bi-Lipschitz. Since $F$ is a local diffeomorphism on $M \backslash\left(f_{\varepsilon}^{-1}(0) \cup\{q\}\right), F$ has no singular points on $M \backslash\left(f_{\varepsilon}^{-1}(0) \cup\{q\}\right)$. Let $F^{+}$and $F^{-}$be the smooth extensions of $\left.F\right|_{\overline{D_{\varepsilon}(p)}}$ and $\left.F\right|_{\overline{D_{\varepsilon}(q)} \backslash\{q\}}$, respectively. Since $\partial F(x)=$ $\operatorname{Conv}\left(\left\{d F_{x}^{+}, d F_{x}^{-}\right\}\right)$for each $x \in f_{\varepsilon}^{-1}(0)$, it follows from Lemma 4.2 that any element in $\partial F(x)$ is of maximal rank. Hence, $F$ has no singular points on $M \backslash\{q\}$. Since $B_{R}\left(f^{-1}(0)\right)$ is open in $M_{1}$ and $f_{\varepsilon}^{-1}(0) \subset B_{R}\left(f^{-1}(0)\right)$, there exists $r \in(0, R)$ such that $B_{r}\left(f_{\varepsilon}^{-1}(0)\right) \subset B_{R}\left(f^{-1}(0)\right)$. Note that $p, q \notin B_{r}\left(f_{\varepsilon}^{-1}(0)\right)$. Let $\varphi$ be a smooth function on $M$ satisfying $0 \leq \varphi \leq 1$ on $M, \varphi \equiv 1$ on $\overline{B_{r}\left(f_{\varepsilon}^{-1}(0)\right)}$, and $\operatorname{supp} \varphi \subset B_{R}\left(f^{-1}(0)\right)$. Define the map $G_{\varepsilon}: M \longrightarrow \mathbb{R}^{n+1}$ by $G_{\varepsilon}:=(1-\varphi) F+\varphi F_{\varepsilon}$, where $F_{\varepsilon}: M \longrightarrow \mathbb{R}^{n+1}$ denotes the smooth approximation of $F$ defined by (2.16). It is clear that

$$
G_{\varepsilon}= \begin{cases}F_{\varepsilon} & \text { on } \overline{B_{r}\left(f_{\varepsilon}^{-1}(0)\right)}, \\ F & \text { on } M \backslash \operatorname{supp} \varphi .\end{cases}
$$

Since $F$ has no singular points on $\overline{B_{r}\left(f_{\varepsilon}^{-1}(0)\right)}$, by the same argument as the proof of Lemma 2.25, $d F_{\varepsilon}$ is injective on $B_{r}\left(f_{\varepsilon}^{-1}(0)\right)$. Since $\varepsilon>0$ is sufficiently small, by (4.1), we can assume that $\left|f_{\varepsilon}-f\right| \leq r$ on $M$. Then, since $F$ is smooth on $B_{R}\left(f^{-1}(0)\right) \backslash \overline{B_{r}\left(f_{\varepsilon}^{-1}(0)\right)}$, $F_{\varepsilon}$ uniformly converges to $F$ on $\overline{B_{R}\left(f^{-1}(0)\right)} \backslash B_{r}\left(f_{\varepsilon}^{-1}(0)\right)$ as $\varepsilon \downarrow 0$ in the $C^{1}$-topology. Thus, we see that $G_{\varepsilon}$ uniformly converges to $F$ on $\overline{B_{R}\left(f^{-1}(0)\right)} \backslash B_{r}\left(f_{\varepsilon}^{-1}(0)\right)$ as $\varepsilon \downarrow 0$ in the $C^{1}$-topology. Hence, $G_{\varepsilon}: M \longrightarrow \mathbb{R}^{n+1}$ is a bi-Lipschitz homeomorphism which is a local diffeomorphism on $M \backslash\{q\}$. Define the map $\psi_{\varepsilon}: M \longrightarrow S^{n}(1)$ by $\psi_{\varepsilon}:=\pi_{S^{n}(1)} \circ G_{\varepsilon}$, where $\pi_{S^{n}(1)}: \mathbb{R}^{n+1} \backslash\{o\} \longrightarrow S^{n}(1)$ denotes the distance projection. By a similar argument to the proof of the differentiable exotic sphere theorem, we see that $\psi_{\varepsilon}$ is a covering map from $M$ onto $S^{n}(1)$. Since $S^{n}(1)$ is simply connected, $\psi_{\varepsilon}$ is injective. Therefore, $\psi_{\varepsilon}$ is a bi-Lipschitz homeomorphism from $M$ onto $S^{n}(1)$ which is a diffeomorphism except for $q$.

Finally, we prove (T-3): Define the map $\widetilde{F}: \mathbb{B}_{\pi}\left(o_{q}\right) \longrightarrow T_{S} S^{n}(1)$ by $\widetilde{F}:=\exp _{S}^{-1} \circ F \circ$ $\exp ^{g_{-}}$. By a similar argument to the proof of the differentiable exotic sphere theorem, we then see that $\widetilde{F}(t v)=t \sigma_{S}^{N} \circ I \circ\left(\sigma_{q}^{p}\right)^{-1}(v)$ for all $(t, v) \in[0, \pi] \times \mathbb{S}_{q}^{n-1}$. Here, $\sigma_{S}^{N}$ denotes the diffeomorphism defined by (1.2). In particular, $\widetilde{F}$ satisfies (3.14). Hence, by Lemma 3.7. $\partial F(q)=\operatorname{Conv}\left(\left\{A_{v} \mid v \in \mathbb{S}_{q}^{n-1}\right\}\right)$, where $A_{v}$ is as in the lemma. For a geodesic segment $\gamma:[0, \pi] \longrightarrow \mathbb{S}_{q}^{n-1}$, let $c:[0, \pi] \longrightarrow \mathbb{S}_{S}^{n-1}$ be the curve defined by $c:=\sigma_{S}^{N} \circ I \circ\left(\sigma_{q}^{p}\right)^{-1} \circ \gamma$. Now, we assume $\operatorname{Lip}^{\mathrm{b}}\left(\left(\sigma_{q}^{p}\right)^{-1}\right)$ satisfies (1.6) for all geodesic segments $\gamma([0, \pi]) \subset \mathbb{S}_{q}^{n-1}$ with $\|\dot{\gamma}\| \equiv 1$. Since

$$
\operatorname{Lip}^{\mathrm{b}}\left(\sigma_{S}^{N} \circ I \circ\left(\sigma_{q}^{p}\right)^{-1}\right)=\operatorname{Lip}^{\mathrm{b}}\left(\left(\sigma_{q}^{p}\right)^{-1}\right),
$$

$\operatorname{Lip}^{\mathrm{b}}\left(\sigma_{S}^{N} \circ I \circ\left(\sigma_{q}^{p}\right)^{-1}\right)$ satisfies (1.6). By Corollary 3.11, $q$ is non-singular for $F$, and hence $F$ has no singular points on $M$. Therefore, it follows from Theorem 1.3 that $M$ and $S^{n}(1)$ 
are diffeomorphic. In the case where (1.7) or (1.10) holds, we can prove that $M$ and $S^{n}(1)$ are diffeomorphic by the same arguments as Sect.3.3.

Remark 4.3 By applying [37, Proposition C] to the two $\operatorname{discs} \overline{D_{\varepsilon}(p)}$ and $\overline{D_{\varepsilon}(q)}$ in the proof above successively, we get a new Riemannian metric on $M$, points $p_{1} \in D_{\varepsilon}(p)$ and $q_{1} \in D_{\varepsilon}(q)$ such that all geodesics (with this new metric) emanating from $p_{1}$ (respectively $q_{1}$ ) pass through the boundary of $\overline{D_{\varepsilon}(p)}$ (respectively $\overline{D_{\varepsilon}(q)}$ ) perpendicularly. Therefore, any geodesic emanating from $p_{1}$ passes through $q_{1}$, i.e., the cut locus of $p_{1}$ consists of $q_{1}$.

Acknowledgements. The authors express their sincere thanks to Professors K. Grove, P. Petersen, and F. Wilhelm, who pointed out a mistake in the first version, back then entitled "A sufficient condition for a pair of bi-Lipschitz homeomorphic manifolds to be diffeomorphic and sphere theorems", of this article. Thanks to the indication, they could arrive at the differentiable exotic sphere theorem. The first named author would like to thank Professor T. Shioya, who suggested the shorter proof of Lemma 2.26 than that in the first version. He is deeply grateful to Professor F.H. Clarke for his encouragement, to Professors M. Gromov, S. Ohta, and T. Yamaguchi for their comments on the first one, and to Professors Y. Agaoka, M. Ishida, and K. Yasui for their having given knowledge about exotic structures, which has had a big influence on the structure of Sect.1. Finally, they thank the referees cordially for careful reading of the manuscript and for helpful and valuable comments on it, which have improved the presentation of this article.

\section{References}

[1] S. Akbulut, Cappell-Shaneson homotopy spheres are standard, Ann. of Math. (2) 171 (2010), no. 3, 2171-2175

[2] A.L. Besse, Manifolds all of whose Geodesics are Closed, Ergeb. Math. Grenzgeb. 93, Springer-Verlag, Berlin-New York, 1978.

[3] J. Cerf, Sur les difféomorphismes de la sphère de dimension trois $\left(\Gamma_{4}=0\right)$, LNM 53 (1968), Springer-Verlag.

[4] J. Cheeger and T.H. Colding, On the structure of spaces with Ricci curvature bounded below. I, J. Differential Geom. 46 (1997), no. 3, 406-480.

[5] F.H. Clarke, Generalized gradients and applications, Trans. Amer. Math. Soc. 205 (1975), 247-262.

[6] F.H. Clarke, On the inverse function theorem, Pacific J. Math. 64 (1976), 97-102.

[7] F.H. Clarke, Optimization and Nonsmooth Analysis, Canadian Mathematical Society Series of Monographs \& Advanced Texts, John Wiley \& Sons Inc., New York, 1983.

[8] F.H. Clarke, Functional Analysis, Calculus of Variations and Optimal Control, GTM 264, Springer, 2013. 
[9] S.K. Donaldson and D.P.Sullivan, Quasiconformal 4-manifolds, Acta Math. 163 (1989), 181-252.

[10] C. Durán, Pointed wiedersehen metrics on exotic spheres and diffeomorphisms of $S^{6}$, Geom. Dedicata 88 (2001), 199-210.

[11] Y. Eliashberg, Contact 3-manifolds twenty years since J. Martinet's work, Ann. Inst. Fourier, Grenoble 42 (1992), 165-192.

[12] M. Freedman, R. Gompf, S. Morrison, and K. Walker, Man and machine thinking about the smooth 4-dimensional Poincaré conjecture, Quantum Topol. 1 (2010), no. 2, 171-208.

[13] R. Gompf, More Cappell-Shaneson spheres are standard, Algebr. Geom. Topol. 10 (2010), no. 3, 1665-1681.

[14] D. Gromoll and W. Meyer, An exotic sphere with nonnegative sectional curvature, Ann. of Math. (2) 100 (1974), 401-406.

[15] M. Gromov, Curvature, diameter and Betti numbers, Comment. Math. Helv. 56 (1981), 179-195.

[16] M. Gromov, Metric Structures for Riemannian and non-Riemannian Spaces, Birkhäuser, Boston, MA, 1999.

[17] K. Grove and H. Karcher, How to conjugate $C^{1}$-close group actions, Math. Z. (2) 132 (1973), 11-20.

[18] K. Grove and K. Shiohama, A generalized sphere theorem, Ann. of Math. (2) 106 (1977), 201-211.

[19] K. Grove and F. Wilhelm, Metric constraints on exotic spheres via Alexandrov geometry, J. Reine Angew. Math. 487 (1997), 201-217.

[20] J.A. Jaramillo, Ó. Madiedo, and L.S. Sánchez-González, Global inversion of nonsmooth mappings on Finsler manifolds, J. Convex Anal. 20 (2013), no. 4, 1127-1146.

[21] H. Karcher, Riemannian center of mass and mollifier smoothing, Comm. Pure Appl. Math., 30 (1977), 509-541.

[22] M. Kervaire and J. Milnor, Groups of homotopy spheres. I, Ann. of Math. (2) 77 (1963), 504-537.

[23] K. Kondo, Grove-Shiohama type sphere theorem in Finsler geometry, Osaka J. Math. 52 (2015), 1143-1162.

[24] K. Kondo and S. Ohta, Topology of complete manifolds with radial curvature bounded from below, Geom. Funct. Anal. 17 (2007), no. 4, 1237-1247.

[25] J. Milnor, On manifolds homeomorphic to the 7-sphere, Ann. of Math. (2) 64 (1956), 399-405. 
[26] J. Munkres, Differentiable isotopies on the 2-sphere, Michigan Math. J. 7 (1960), 193-197.

[27] Y. Otsu, K. Shiohama, and T. Yamaguchi, A new version of differentiable sphere theorem, Invent. Math. 98 (1989), 219-228.

[28] P.Petersen and F. Wilhelm, An exotic sphere with positive sectional curvature, arXiv:0805.0812.

[29] H. Rademacher, Über partielle und totale differenzierbarkeit von Funktionen mehrerer Variabeln und über die Transformation der Doppelintegrale, Math. Ann. 79 (1919), 340-359.

[30] L. Rifford, A Morse-Sard theorem for the distance function on Riemannian manifolds, Manuscripta Math. 113 (2004), no. 2, 251-265.

[31] Y. Shikata, On a distance function on the set of differentiable structures, Osaka J. Math. 3 (1966), 65-79.

[32] Y.Shikata, On the differentiable pinching problem, Osaka J. Math. 4 (1967), 279287.

[33] I.M. Singer and J.A. Thorpe, Lecture Notes on Elementary Topology and Geometry, UTM, Springer, 1976.

[34] S. Smale, Generalized Poincaré's conjecture in dimensions greater than four, Ann. of Math. 74 (1961), 391-406.

[35] S. Smale, On the structure of manifolds, Amer. J. Math. 84 (1962), 387-399.

[36] J.R. Stallings, Polyhedral homotopy-spheres, Bull. Amer. Math. Soc. 66 (1960), 485488.

[37] A.D. Weinstein, The cut locus and conjugate locus of a riemannian manifold, Ann. of Math. (2) 87 (1968), 29-41.

[38] J.H.C. Whitehead, On the covering of a complete space by the geodesics through a point, Ann. of Math. (2) 36 (1935), 679-704.

[39] K. Yasui, Nonexistence of Stein structures on 4-manifolds and maximal ThurstonBennequin numbers, to appear in J. Symplectic Geom.

[40] E.C.Zeeman, The Poincaré conjecture for $n \geq 5,1962$ Topology of 3-manifolds and related topics (Proc. The Univ. of Georgia Institute, 1961), 198-204, Prentice-Hall, Englewood Cliffs, N.J. 
K. Kondo

Department of Mathematical Sciences, Yamaguchi University

Yamaguchi City, Yamaguchi Pref. 753-8512, Japan

e-mail: keikondo@yamaguchi-u.ac.jp

M. Tanaka

Department of Mathematics, Tokai University

Hiratsuka City, Kanagawa Pref. 259-1292, Japan

e-mail: tanaka@tokai-u.jp 